\title{
TOI-1278 B: SPIRou Unveils a Rare Brown Dwarf Companion in Close-in Orbit around an M Dwarf
}

Étienne Artigau ${ }^{1,2}\left(10\right.$, Guillaume Hébrard ${ }^{3}$ (1) , Charles Cadieux $^{1,2}$, Thomas Vandal ${ }^{1,2}$, Neil J. Cook ${ }^{1,2}$ (1) René Doyon $^{1,2}$, Jonathan Gagne $^{1,4}$, Claire Moutou ${ }^{5}$, Eder Martioli ${ }^{3,6}$, Antonio Frasca ${ }^{7}$, Farbod Jahandar ${ }^{1}(\mathbb{1})$, David Lafrenière ${ }^{1}$, Lison Malo ${ }^{1,2}$ (i),

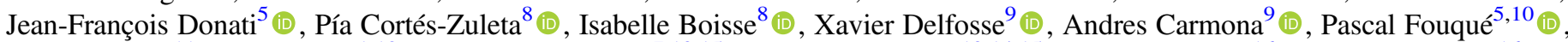
Julien Morin $^{11}$, Jason Rowe ${ }^{1,12}$ (10), Giuseppe Marino ${ }^{7,13,15}$ (D) Riccardo Papini $^{13,14,15}$, David R. Ciardi ${ }^{16}$, Michael B. Lund ${ }^{16}$ (D), Jorge H. C. Martins ${ }^{17}$, Stefan Pelletier ${ }^{1}$, Luc Arnold ${ }^{10}$ (i), François Bouchy ${ }^{18}$ (D), Thierry Forveille ${ }^{9}$ (i), Nuno C. Santos ${ }^{19,20}$ (D), Xavier Bonfils $^{9}$ (1), Pedro Figueira ${ }^{21,22}$ (1), Michael Fausnaugh ${ }^{23}$, George Ricker ${ }^{24,25}$ (1), David W. Latham ${ }^{26}$, Sara Seager ${ }^{24,27,28}$ (i), Joshua N. Winn ${ }^{29}$, Jon M. Jenkins ${ }^{30}$ (1) , Eric B. Ting ${ }^{30}$, Guillermo Torres ${ }^{26}$, and João Gomes da Silva ${ }^{19}$ (i)

${ }^{1}$ Institute for Research on Exoplanets, Université de Montréal, Département de Physique, C.P. 6128 Succ. Centre-ville, Montréal, QC H3C 3J7, Canada etienne.artigau@umontreal.ca

${ }^{2}$ Observatoire du Mont-Mégantic, Université de Montréal, Département de Physique, C.P. 6128 Succ. Centre-ville, Montréal, QC H3C 3J7, Canada

${ }^{3}$ Sorbonne Université, CNRS, UMR 7095, Institut dAstrophysique de Paris, 98 bis bd Arago, F-75014 Paris, France

${ }^{4}$ Planétarium Rio Tinto Alcan, Espace pour la Vie, 4801 av. Pierre-de Coubertin, Montréal, Québec, Canada ${ }^{5}$ Univ. de Toulouse, CNRS, IRAP, 14 Avenue Belin, F-31400 Toulouse, France

${ }^{6}$ Laboratório Nacional de Astrofísica, Rua Estados Unidos 154, Itajubá, MG 37504-364, Brazil

${ }^{7}$ INAF-Osservatorio Astrofisico di Catania, Via S. Sofia 78, I-95123 Catania, Italy

${ }^{8}$ Aix Marseille Univ, CNRS, CNES, LAM, Marseille, France

${ }^{9}$ Univ. Grenoble Alpes, CNRS, IPAG, F-38000 Grenoble, France

${ }^{10}$ Canada-France-Hawaii Telescope, CNRS, Kamuela, HI 96743, USA

${ }^{11}$ Université de Montpellier, CNRS, LUPM, F-34095 Montpellier, France

12 Bishops University, 2600 College Street, Sherbrooke, QC J1M 1Z7, Canada

${ }^{13}$ Wild Boar Remote Observatory, San Casciano in val di Pesa, Firenze, I-50026 Italy

${ }^{14}$ American Association of Variable Star Observers, 49 Bay State Road, Cambridge, MA 02138, USA

${ }^{15}$ Gruppo Astrofili Catanesi, Catania, I-95128, Italy

${ }^{16}$ NASA Exoplanet Science Institute-Caltech/IPAC 1200 E. California Boulevard, Pasadena CA, 91125, USA

${ }^{17}$ Instituto de Astrofísica e Ciências Espaciais, Universidade do Porto, Rua das Estrelas, Porto, Portugal

${ }^{18}$ Departement dastronomie, Université de Genève, Chemin des Maillettes 51, CH-1290 Versoix, Switzerland

${ }^{19}$ Instituto de Astrofísica e Ciências do Espaço, Universidade do Porto, CPAU, Rua das Estrelas, 4150-762 Porto, Portugal

${ }^{20}$ Departamento de Física e Astronomia, Faculdade de Ciências, Universidade do Porto, Rua do Campo Alegre, 4169-007 Porto, Portugal

${ }^{21}$ European Southern Observatory, Alonso de Cordova 3107, Vitacura, Santiago, Chile

${ }^{22}$ Instituto de Astrofísica e Ciências do Espaço, Universidade do Porto, CAUP, Rua das Estrelas, 4150-762 Porto, Portugal

${ }^{23}$ Department of Physics and Kavli Institute for Astrophysics and Space Research, Massachusetts Institute of Technology, Cambridge, MA 02139, USA

${ }^{24}$ MIT Kavli Institute for Astrophysics and Space Research, Massachusetts Institute of Technology, Cambridge, MA 02139, USA

${ }_{25}^{25}$ MIT Department of Physics, Massachusetts Institute of Technology, Cambridge, MA 02139, USA

${ }^{26}$ Center for Astrophysics | Harvard \& Smithsonian, 60 Garden Street, Cambridge, MA 02138, USA

${ }^{27}$ Earth and Planetary Sciences, Massachusetts Institute of Technology, 77 Massachusetts Avenue, Cambridge, MA 02139, USA

${ }^{28}$ Department of Aeronautics and Astronautics, MIT, 77 Massachusetts Avenue, Cambridge, MA 02139, USA

${ }^{29}$ Department of Astrophysical Sciences, Princeton University, 4 Ivy Lane, Princeton, NJ 08544, USA

${ }^{30}$ NASA Ames Research Center, Moffett Field, CA 94035, USA

Received 2021 March 16; revised 2021 June 7; accepted 2021 June 7; published 2021 September 16

\begin{abstract}
We present the discovery of an $18.5 \pm 0.5 M_{\text {Jup }}$ brown dwarf (BD) companion to the M0V star TOI-1278. The system was first identified through a percent-deep transit in Transiting Exoplanet Survey Satellite photometry; further analysis showed it to be a grazing transit of a Jupiter-sized object. Radial velocity (RV) follow-up with the SPIRou near-infrared high-resolution velocimeter and spectropolarimeter in the framework of the 300-night SPIRou Legacy Survey carried out at the Canada-France-Hawaii Telescope led to the detection of a Keplerian RV signal with a semi-amplitude of $2306 \pm 10 \mathrm{~m} \mathrm{~s}^{-1}$ in phase with the 14.5 day transit period, with a slight but nonzero eccentricity. The intermediate-mass ratio $\left(M_{\star} / M_{\text {comp }} \sim 31\right)$ is unique for having such a short separation $(0.095 \pm 0.001 \mathrm{au})$ among known M-dwarf systems. Interestingly, M-dwarf-BD systems with similar mass ratios exist with separations of tens to thousands of astronomical unit.
\end{abstract}

Key words: Brown dwarfs - Radial velocity - Transit photometry - Transits - M dwarf stars

\section{Introduction}

Over the past $25 \mathrm{yr}$, the search for and characterization of planets around other stars has moved from speculative to one of the most active fields in astronomy. Among planetary systems, those orbiting the coolest stars, $\mathrm{M}$ dwarfs, are of particular interest for a number of reasons. They provide insight into planetary formation in a regime of host-star mass that differs significantly from our own solar system, with some systems more reminiscent in their architecture and scale to the Galilean moon system. Furthermore, as $\mathbf{M}$ dwarfs have radii and masses that are significantly smaller than those of our Sun (M0V to M9V dwarfs ranging, respectively, from 0.6 to $0.1 R_{\odot}$ and 0.6 to $0.1 M_{\odot}$ ), planets are generally easier to discover and characterize through transits than similarly sized planets around sunlike stars. This is particularly true for older, slowly rotating $\mathrm{M}$ dwarfs, with activity being a limiting factor for a number of 
low-mass stars. Furthermore, the lower luminosity of M dwarfs (0.1 to $10^{-3.5} L_{\odot}$; Veeder 1974; Faherty et al. 2016) implies that the habitable zone (HZ) is much closer to the host star than in our solar system, implying that the orbits of planets in the $\mathrm{HZ}$ can be sampled on timescales of days. Given that $\mathrm{M}$ dwarfs make up the majority of stars among nearby stellar systems (being five times more numerous than FGK stars), it is likely that most of the $\mathrm{HZ}$ planets within $10 \mathrm{pc}$ are found around M dwarfs (Figueira et al. 2016). This is exemplified by our closest stellar neighbor, the M5.5V Proxima Centauri, having a terrestrial planet in the HZ (Anglada-Escudé et al. 2016).

As they provide an easier path to the characterization of $\mathrm{HZ}$ terrestrial planets, planets around $M$ dwarfs have been the subject of considerable efforts. Transit searches dedicated to or optimized for M dwarfs (Irwin et al. 2009) have been successful in identifying systems such as the seven Earthsized planets around TRAPPIST-1 (Gillon et al. 2017), and the ongoing Transiting Exoplanet Survey Satellite (TESS) mission has a top-level Requirement of uncovering 1000 small $\left(R_{\mathrm{p}}<4 R_{\oplus}\right)$ planets and measuring the masses of 50 such planets (Ricker et al. 2014). This requirement is not restricted to $\mathrm{M}$ dwarfs, but the TESS bandpass was designed to extend significantly to the red to maximize its sensitivity to planets around $\mathrm{M}$ dwarfs, compared to the Kepler mission, which had a bluer bandpass better matched to sunlike stars. While Earth-sized HZ planets receive significant attention, a number of statistical properties of the M-dwarf planets have emerged recently regarding more massive companions to M dwarfs.

Jupiter-mass planets are remarkably rare around $\mathrm{M}$ dwarfs at short separations. While $0.4 \%-1 \%$ of sunlike stars host a hot Jupiter (occurrence rate estimates differ depending on detection method considered; Wang et al. 2015), they are rarer around M dwarfs (Meyer et al. 2018); although, a few systems have recently been discovered (e.g., Bayliss et al. 2018). This is most unlikely to be an observational bias, as a hot Jupiter around an $\mathrm{M}$ dwarf would induce $\mathrm{a}>100 \mathrm{~m} \mathrm{~s}^{-1}$ radial velocity (RV) signal (from Kepler's laws, combining a $1 M_{\text {Jup }}$ on a 4 day orbit around a $0.1-0.5 M_{\odot}$ host) and a readily detectable transit ( $\sim 4 \%$ to $\sim 100 \%$ deep for M0V to M9V; from radius relations in Boyajian et al. 2012; Filippazzo et al. 2015).

Here, we report the discovery of an $18.5 M_{\text {Jup }}$ transiting brown dwarf (BD) companion on a 14.5 day orbit around the M0V star TOI-1278 (see Table 1 for basic properties of the host star), above the maximum mass expected to form through a protoplanetary disk; typically $<1 \quad M_{\text {Jup }}$ with only a few objects between 1 and $10 M_{\text {Jup }}$ in the 90-star sample by Ansdell et al. (2016). It occupies a nearly empty part of the mass distribution of close-in companions of $\mathrm{M}$ dwarfs, between the Jupiter population and the massive BD companions. In Section 2 we present discovery and follow-up observations of the system. In Section 3 we assess the properties of the host star, and in Section 4 we detail the data analysis. Finally, in Section 5 we discuss the properties of the system in comparison to other planetary and BD companions, its dynamical fate, and characterization prospects.

\section{Observations}

We present below the TESS discovery data of TOI-1278, as well as its ground-based follow-up observations in photometry, high-resolution imaging, low-resolution spectroscopy, and precise near-infrared (NIR) velocimetry, all required to establish the nature of the transiting object TOI-1278 B.

\subsection{TESS Photometry}

TOI-1278 was observed by TESS (Ricker et al. 2014) in sector 15 with CCD 4 on Camera 1 from 2019 August 15 to September 11. This target was selected in the Cool Dwarf List (Muirhead et al. 2018), a specially curated list of late-K and $M$ dwarfs included in the TESS Input Catalog (TIC; Stassun et al. 2018, 2019) and the Candidate Target List for 2 minute cadence light-curve sampling. We used the publicly available ${ }^{31}$ Presearch Data Conditioning Simple Aperture Photometry (PDCSAP; Smith et al. 2012; Stumpe et al. 2012, 2014) lightcurve produced by the NASA Ames Science Processing Operations Center (SPOC; Jenkins et al. 2016). The PDCSAP flux values are corrected for long-term systematic trends seen among other stars in the same sector/camera/CCD using Cotrending Basis Vectors. No long-term stellar variability on timescales shorter than the time series considered here are removed. Furthermore, these PDCSAP fluxes account for the dilution caused by nearby stars. The TIC contamination ratio defined as the flux from other sources within the aperture divided by the target star flux (in TESS-band) is 0.326395 for TOI-1278. This dilution correction is important, as any other flux contribution would lead to an underestimation of the transiting object radius by measuring a smaller transit depth. The TOI-1278 normalized PDCSAP light curve is presented in Figure 1 and clearly shows two transit events.

SPOC sector 15 data validation (DV) reports (Twicken et al. 2018; Li et al. 2019) first identified the two transits with a signal-to-noise ratio $(\mathrm{S} / \mathrm{N})$ of 16.5 . Both events were reported to be $\mathrm{V}$-shaped, suggestive of a high impact parameter, with an average depth of $1.03 \% \pm 0.07 \%$. This led to the announcement of planet candidate TOI-1278.01 with an orbital period of 14.476 days and an estimated radius of $9.3 R_{\oplus}$. Three consecutive transit detections are usually needed to confirm the previous two were produced by the same object. In this case, the two transits were found to have consistent duration, suggesting that they are caused by the same object rather than two planets within the same system or two planets orbiting different stars. We furthermore inspected the PDCSAP light curve near phase $\phi_{\mathrm{sec}}=0.496$, the predicted phase of secondary eclipse based on the geometry of the TOI-1278 B orbit constrained in Section 4. The eclipse signal due to reflected light is estimated at $13 \mathrm{ppm}$ for a geometric albedo of 0.5 . Given TESS photometric precision, no secondary eclipse is detected, and we can only infer a $3 \sigma$ upper limit of $840 \mathrm{ppm}$ for the eclipse depth.

\subsection{Ground-based Transit Monitoring}

While the position of TOI-1278 in the HR diagram (see Section 3) strongly suggests that the star is not itself an equalluminosity eclipsing binary, the TESS detection could still be attributed to a nearby eclipsing binary (NEB) located within a few pixels. This is due to TESS's coarse image sampling ( $21^{\prime \prime}$ per pixel) and, hence, the need to confirm on-target transit events with arcsecond angular resolution ground-based observations. In the case of TOI-1278, additional transit

\footnotetext{
${ }^{31}$ Mikulski Archive for Space Telescopes: https://archive.stsci.edu/tess/.
} 

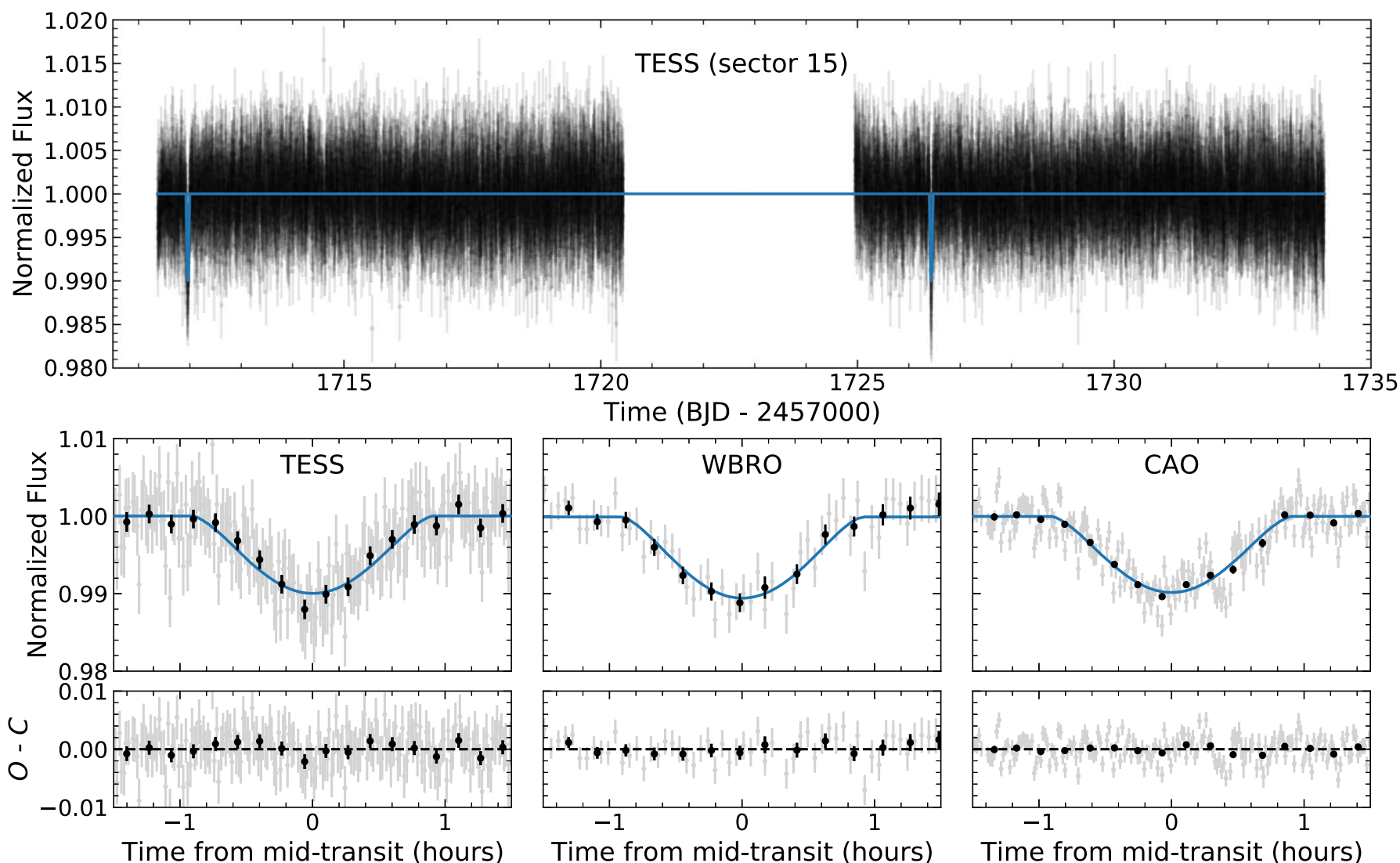

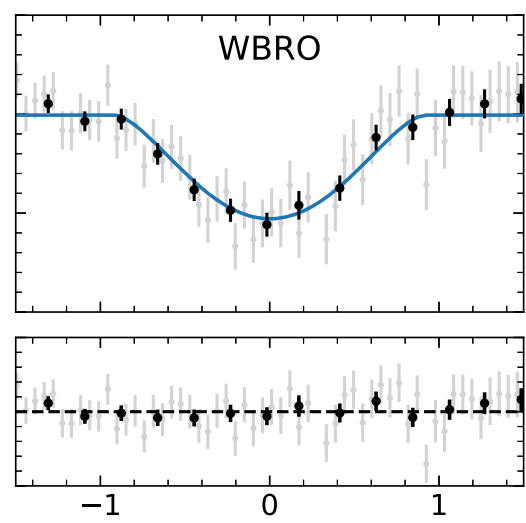

Time from mid-transit (hours)
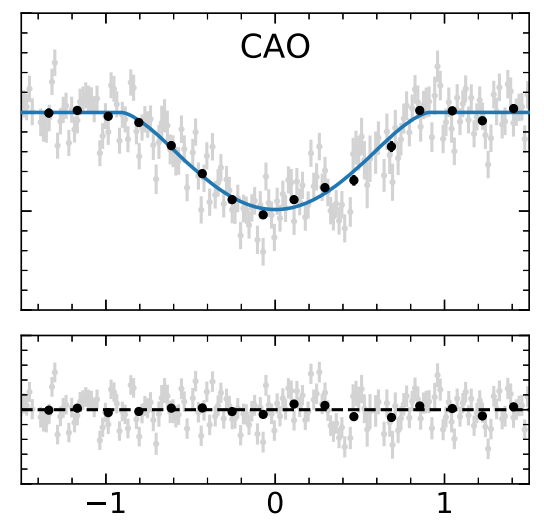

Time from mid-transit (hours)

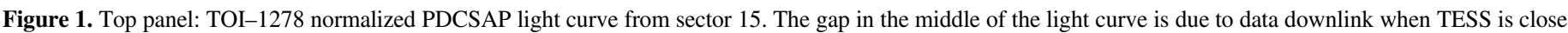

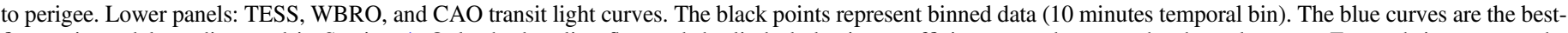

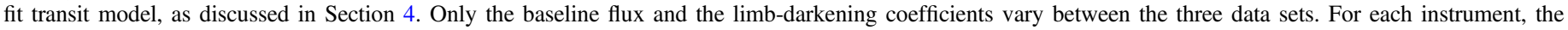
residuals (observed - calculated) are shown below the transit. The TESS data set is phase-folded and covers two transits.

observations were crucial to set stronger constraints on its lightcurve determination, particularly on the orbital period $P$, the radius of the transiting object $R_{\mathrm{p}}$, and the impact parameter $b$. Ground-based monitoring was spurred by the TESS discovery and was obtained to further constrain transit properties. We did not obtain a full out-of-transit light curve.

We scheduled two transit observations using the TESS Transit Finder (TTF), a customized version of the Tapir software package (Jensen 2013). We first observed TOI-1278 at Wild Boar Remote Observatory (WBRO) near Florence, Italy on 2019 October 27 without any filter (clear). A grazing, percent-deep, transit was detected on-target using a 4!"7 aperture and was on time given the TTF prediction and uncertainty. The second observation was conducted at M.G. Fracastoro station (Mt. Etna, $1735 \mathrm{~m}$ a.s.l.) of the Catania Astrophysical Observatory (CAO), in Italy on 2019 November 25 in $I_{\mathrm{C}}$ with the $0.91 \mathrm{~m}$ telescope. Using a 5."3 aperture, a second V-shaped transit of the same depth was detected at the expected time. Both data sets were consistent with TESS in transit depth, shape, duration, and timing. Ground-based photometric follow-ups significantly increased the time baseline of our data set and further constrained the orbital fit. We have thus included these additional photometric observations, together with TESS transits, in our joint model described in Section 4. Calibration and light-curve extraction by differential photometry were accomplished using AstroImageJ (AIJ; Collins et al. 2017).

\subsection{Ground-based Photometry}

To constrain the stellar parameters of the host star, in particular the effective temperature, we obtained complementary optical photometry at the $\mathrm{CAO}$, as the values reported in the TIC had relatively large uncertainties. Photometric measurements in $B, V, R_{\mathrm{C}}$, and $I_{\mathrm{C}}$ were obtained on 2020 December 1 (see Table 2). Standard stars in the cluster NGC 7790 (Stetson 2000) were observed just after TOI-1278 to calculate the zero-points and the transformation coefficients to the Johnson-Cousins system. The errors on the $B, V, R_{\mathrm{C}}$, and $I_{\mathrm{C}}$ magnitudes include both the photometric uncertainty coming from the photon statistics and the error of standardization based on the NGC 7790 data.

\subsection{High-resolution Imaging}

We searched for sources within a few arcseconds of TOI1278 with Palomar/PHARO NIR adaptive optics imaging on 2019 November 9 in the $\operatorname{Br} \gamma$ band. As shown in Figure 2, no companion is detected at $5 \sigma$ with a contrast ratio $\Delta \mathrm{Br} \gamma \leqslant 7.0 \mathrm{mag}$ for separation greater than $1^{\prime \prime}(76 \mathrm{au})$ and $\Delta \mathrm{Br} \gamma \leqslant 6.335 \mathrm{mag}$ for separation over 0.5 (38 au). These contrasts thus exclude a companion brighter than $M_{K} \sim 11.7$, corresponding to an $\mathrm{L} 4$ spectral type and $L_{\mathrm{bol}}=10^{-4.1} L_{\odot}$ for field objects (using polynomial relations in Faherty et al. 2016). At an age $>1$ Gyr (see Section 5), this rules out stellar companions down to the upper limit of the BD regime (Phillips et al. 2020 models). 


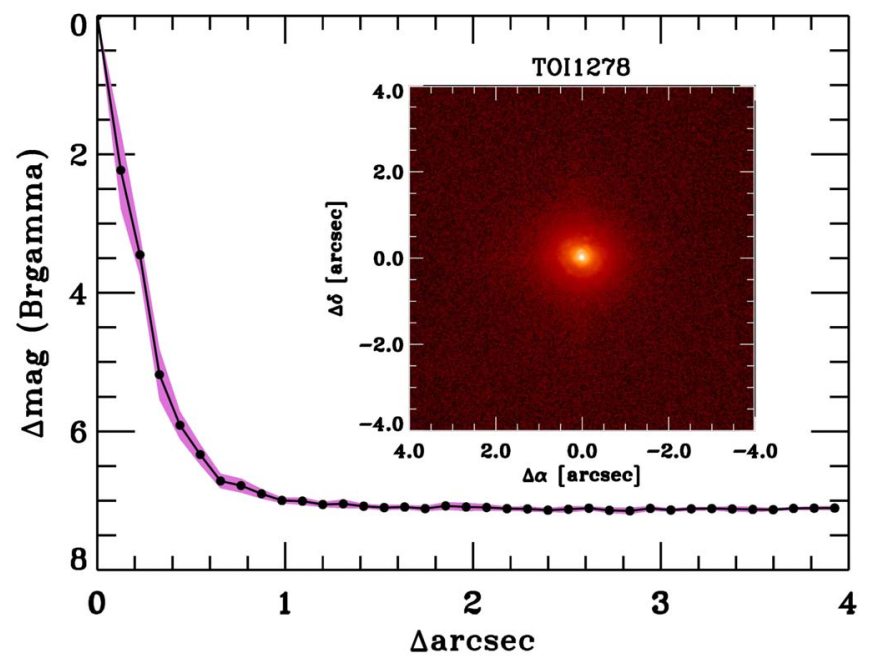

Figure 2. $\operatorname{Br} \gamma 5 \sigma$ contrast curve of TOI-1278 from Palomar/PHARO NIR adaptive optics imaging. No close companion is detected.

\subsection{SPIRou Velocimetry}

TOI-1278 was observed over 12 epochs from 2020 May 31 to November 1 using SPIRou, the NIR $(0.98-2.5 \mu \mathrm{m})$ spectropolarimeter installed on the Canada-France-Hawaii Telescope (Donati et al. 2018, 2020). The data set was obtained as part of the ongoing SPIRou Legacy Survey (SLS; Donati et al. 2020).

At each epoch, a four-measurement polarimetric sequence was obtained, each measurement having a 5 min integration, except for June 7, when eight measurements were obtained due to poorer observing conditions. Per-exposure S/Ns of 20-38 were obtained for the middle of the $H$ band. These $\mathrm{S} / \mathrm{N}$ values are for a spectral sampling of the SPIRou data of $\sim 2.2 \mathrm{~km} \mathrm{~s}^{-1}$ pixel $^{-1}$. We rejected a handful of observations due to low $\mathrm{S} / \mathrm{N}$, resulting in 47 usable precision radial velocity (PRV) measurements at 10 different epochs. Table A1 compiles perepoch radial velocity and associated uncertainties used in this analysis.

Observations were reduced with the standard data reduction pipeline for SPIRou (APERO, version 0.6.131; Cook et al. in prep). APERO automatically handles all reduction steps necessary for PRV. In brief, the pipeline first corrects detector-related effects (specific to H4RG infrared arrays, i.e., the capacitive coupling between amplifiers, the $1 / \mathrm{f}$ noise, and the dark current; Artigau et al. 2018a). Then, APERO locates the bad pixel positions, the exact positions of orders on the array, and determines the shape of the instrument slicer (Micheau et al. 2018) as well as determining the flat and blaze corrections to apply, using nightly calibration sequences. The calibrated data is then optimally extracted (Horne 1986) in both polarization channels, simultaneously $(\mathrm{AB})$ and separately (A and B), as well as the simultaneous calibration channel (C). Extracted two-dimensional (E2DS, 49 orders by 4088 pixels) images are produced, and corrected for thermal emission. As the simultaneous Fabry-Perot calibration was used, leakage from the calibration channel into the science channels was also corrected. Nightly wavelength solutions are constructed using a combination of hollow-cathode and Fabry-Perot calibrations (Cersullo et al. 2019; Hobson et al. 2021). Barycentric corrections were determined within APERO using the barycorrpy package (Kanodia \& Wright 2018).
Telluric and night-sky emission correction is also done automatically using APERO. Night-sky emission is corrected using a principal component analysis (PCA) model of $\mathrm{OH}$ emission constructed from a library of high-S/N sky observations. Telluric absorption correction is done using a PCA-based approach on residuals after fitting for a basic atmospheric transmission model (TAPAS; Bertaux et al. 2014); the details of the PCA approach are described in Artigau et al. (2014), and its implementation is described in APERO (Artigau et al. in prep).

With the telluric-corrected spectrum, we perform an RV measurement using a cross-correlation function (CCF) with a weighted mask of stellar absorption lines. The mask used must be constructed with a star that reasonably matches in temperature TOI-1278. From existing SPIRou Legacy Survey (SLS) data, we have a number of bright, nearby, M dwarfs that have been observed 100 times with an $\mathrm{S} / \mathrm{N}>150$ in the $H$ band. The best match to TOI-1278 was found to be the M0.5V Gl 846, and we use the CCF mask constructed from that spectrum through the PRV analysis.

The CCF is computed for each of the 49 SPIRou orders. Even though the spectra are telluric-corrected, parts of the domain between photometric bandpasses are unusable for PRV. We rejected all orders outside of the $Y J H K$ bandpasses. The effective domain used is $980-1113 \mathrm{~nm}, 1153-1354 \mathrm{~nm}$, 1462-1808 nm, and 1957-2400 nm. Per-order CCFs for usable orders were weighted by the $\mathrm{S} / \mathrm{N}$ combined with their $\mathrm{RV}$ contents defined in Bouchy et al. (2001) and combined in a per-integration $\mathrm{CCF}$. We used a cross-correlation of each observation's CCF with the mean CCF of all observations to measure TOI-1278's velocity. This resulted in a slightly lower dispersion of RV than fitting a Gaussian model to each individual CCF. No method that attempted to measure the velocity from the CCF profile (Gaussian fit, bisector mean position, adjustment of the mean CCF to each observation) led to a significant change in the main result: a $K \sim 2300 \mathrm{~m} \mathrm{~s}^{-1}$ signal consistent in phase and with the period of the companion detected through the photometric monitoring (see Figure 3). The per-epoch RV uncertainty was computed by using Equations (6) and (13) in Bouchy et al. (2001) for the individual CCFs. The computed uncertainties are consistent with the point-to-point dispersion within each epoch. To prevent any biases related to underestimated RV errors, we explored the use of an additional jitter term in the RV model described in Section 4. We found that the posterior of the jitter term was consistent with zero, with a $1 \sigma$ upper limit at $8 \mathrm{~m} \mathrm{~s}^{-1}$, and that all other model parameters remained unaffected well within their $1 \sigma$ uncertainty. We therefore conclude that the reported RV uncertainties are accurate, and we do not include the jitter term in the model presented in Section 4.

\subsection{Near-infrared Spectroscopy}

We obtained a low-resolution NIR $(0.70-2.52 \mu \mathrm{m})$ spectrum of TOI-1278 on UT 2020 December 24 with the SpeX spectrograph at the NASA Infrared Telescope Facility (IRTF; Rayner et al. 2003) to improve the wavelength coverage of our spectral energy distribution and refine the estimated radius of TOI-1278. We used the 0 ". 8 slit in prism mode, resulting in a resolving power $\lambda / \Delta \lambda \approx 75$.

Observing conditions were clear with a 0 ". 5 atmospheric seeing. We obtained four $60 \mathrm{~s}$ exposures of TOI-1278 in an ABBA dither pattern along the slit at an airmass of 1.56, 

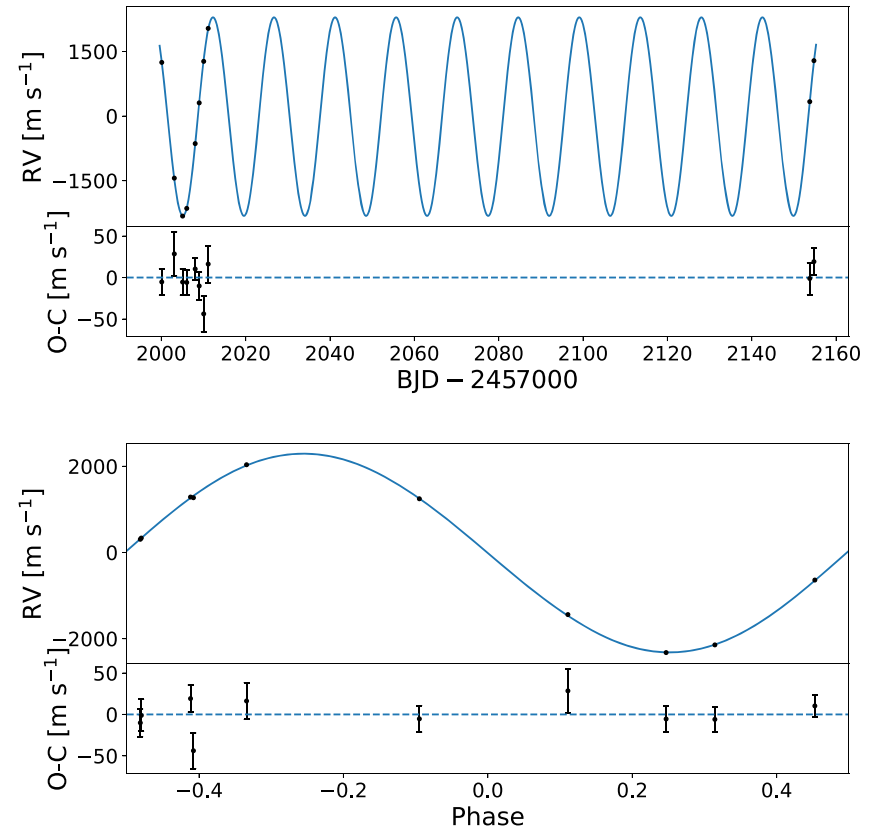

Figure 3. Top panel: RV of TOI-1278 from SPIRou monitoring with an overplot of the best-fit orbital solution. The RV measurements are binned per epoch (typically four observations per night). The median binned error bar is 16.5 $\mathrm{m} \mathrm{s}^{-1}$, and the per-epoch residual (shown below) rms is $19 \mathrm{~m} \mathrm{~s}^{-1}$. The orbital fit is discussed in Section 4. Bottom panel: same as the top panel, but phasefolded using the best-fit orbital period and time of inferior conjunction.

followed by standard SpeX prism calibrations. This was immediately followed by the observation of the A0V-type spectral standard HD 203470 to correct for telluric absorption. We obtained 30 short exposures of HD 203470 in ABBA patterns along the slit at an average airmass of 1.64. We used exposure times in the range $0.5-5 \mathrm{~s}$ to maximize $\mathrm{S} / \mathrm{N}$ while ensuring that we would obtain at least one set of exposures inside the linear range of the improved detector response of the recently upgraded SpeX detector (no sequence reached the nonlinear detector regime).

All data were reduced using SpexTool v4.1 (Cushing et al. 2004). Two-dimensional traces were first extracted using an optimal extraction algorithm, and each resulting onedimensional raw spectrum was visually inspected to remove bad pixel clusters that randomly occur in the two-dimensional frames during long exposures. The raw spectra are then combined in an $\mathrm{S} / \mathrm{N}$ weighted average, and the raw science target is corrected for telluric absorption using the standard xtell cor procedure (Vacca et al. 2003).

\section{Stellar Characterization}

Other than for the presence of a transiting companion (see sections below), TOI-1278 is an unremarkable M dwarf at a distance of $75.46 \pm 0.07 \mathrm{pc}$ (Gaia Collaboration et al. 2021). In the local-neighborhood HR diagram (see Figure 4), TOI-1278 falls onto the main sequence of low-mass stars, suggestive that it is not an equal-luminosity binary nor particularly inflated due to youth. Also notable is the relatively low level of photometric activity of the star; it displayed a flat light curve, other than for the companion transits, at the part-per-thousand level over the month-long monitoring by TESS (see Figure 1). This argues against a young star that would have frequent flaring; such variability on timescales of hours to days would have been preserved by the PDCSAP photometry.

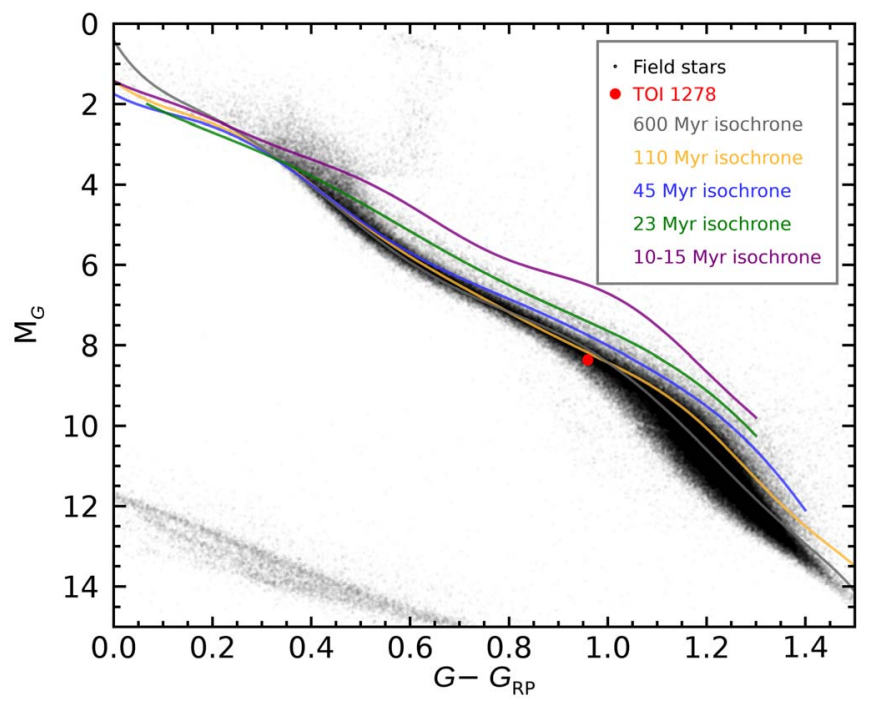

Figure 4. Position of TOI-1278 for the $<100$ pc volume-limited sample based on GAIA parallax and photometric measurements. TOI-1278 falls on the main sequence, and it is significantly offset from empirical tracks for young associations that are $<600 \mathrm{Myr}$ old. The position on the HR diagram also rules out a near-equal-luminosity binary, which would be offset from the main sequence by $0.75 \mathrm{mag}$ (roughly the position of the $45 \mathrm{Myr}$ isochrone). Empirical isochrones are from Gagné et al. (2018) and Gagné et al. (in prep). and are based on the Upper Scorpius, Lower Centaurus Crux and Upper Centaurus associations (10-15 Myr), the $\beta$ Pictoris and 32 Ori associations (23 Myr), TW Hydra, Columba and Carina associations (45 Myr), the Pleiades (110 Myr), and Coma Berenices cluster (600 Myr).

\subsection{Spectral Energy Distribution and Radius}

We used the method of Filippazzo et al. (2015) to estimate the bolometric luminosity of TOI-1278. We used the updated trigonometric parallax from Gaia eDR3 to calibrate all available broadband photometry (listed in Table 2), and our SpeX NIR spectrum was normalized to an error-weighted combination of the predicted absolute fluxes from the broadband photometric measurements that overlap with the spectrum, following Filippazzo et al. (2015). We adopted the effective temperature determined in Section $3.2(3799 \pm 42 \mathrm{~K})$ and assigned a Rayleigh-Jeans and Wien tails of the appropriate blackbody temperature. Linear interpolation in logarithm space was used to construct the spectral energy distribution outside of the SpeX spectrum and between available broadband photometric measurements. Our resulting spectral energy distribution, shown in Figure 5, allowed us to measure a bolometric luminosity of $0.0614 \pm 0.0001 L_{\odot}$. This bolometric luminosity measurement is semi-empirical; one only needs to assume a temperature to set the shape of the Wien tail. Considering that it accounts for a small fraction of the overall flux, the impact of the uncertainty on the effective temperature is much smaller than the uncertainties propagated from photometry and parallax measurements. Regarding the Rayleigh-Jeans regime, the only assumption is that the two reddest photometric data points are well past the peak of the Plank function, free of strong molecular absorption and not significantly affected by a circumstellar disk. Considering that the spectral energy distribution peaks at $\sim 1 \mu \mathrm{m}$ and that the reddest bandpasses are at 4.6 and $11.6 \mu \mathrm{m}$, this assumption is justified. Combining this measurement with our model-dependent $T_{\text {eff }}$ allows us to derive a semi-empirical radius measurement of $0.573 \pm$ $0.012 R_{\odot}$. This measurement is marginally larger (by $5 \% \pm$ $4 \%$ ) than predictions from the solar-metallicity models of 


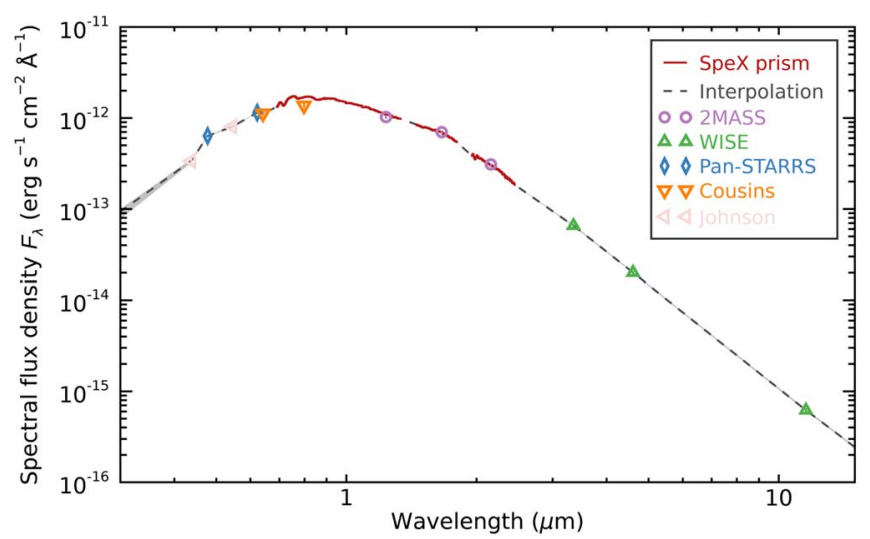

Figure 5. Spectral energy distribution of TOI-1278. Absolute fluxes estimated using photometry from various surveys are displayed as separate symbols, as indicated in the legend. Our NIR SpeX spectrum normalized with absolute fluxes from overlapping photometry is shown as a red solid line. Linear interpolation in logarithm space, and Rayleigh-Jeans and Wien tails of the appropriate effective temperature are shown as dashed lines to complete the spectral energy distribution used in our calculation of the bolometric luminosity.

Baraffe et al. (1998), and only $1 \% \pm 3 \%$ larger than empirical spectral type to radius relations for field stars (Pecaut \& Mamajek 2013). ${ }^{32}$ This radius measurement is also consistent with interferometric measurements for nearby $M$ dwarfs (Demory et al. 2009), which tend to be marginally inflated compared with solar-metallicity models, by about $0 \%-2 \%$. This is in stark contrast with young $\mathrm{M}$ dwarfs, which tend to be significantly inflated (by $150 \%-250 \%$ at 20-40 Myr) compared with theoretical models and older M dwarfs, likely because of their faster rotation rates that drive strong magnetic fields, which in turn affect the hydrostatic balance of young $M$ dwarfs (Malo et al. 2014). This indicates that TOI-1278 is likely not a young $\mathrm{M}$ dwarf, consistent with our small $v \sin i$ measurement (see Section 3.2).

Our SpeX low-resolution NIR spectrum indicates an M0 spectral type when compared with spectral type standards in the IRTF spectral library (Cushing et al. 2005; Rayner et al. 2009), consistent with our best-matching CCF mask from the M0.5Vtype template G1846. Constraining the age of TOI-1278 is key for further analysis of its properties; a young M0V would have an inflated radius and lower mass compared to gigayear-old objects. This would impact both the derived mass from RV measurements and radius estimate from transit properties. Also, uncovering a young transiting BD would be interesting to constrain evolution models.

Rare objects such as the young $\left(93_{-29}^{+61} \mathrm{Myr}\right)$ inflated transiting BD TOI-811 b provide complementary constraints to BD evolutionary models (Carmichael et al. 2020). This provides a complementary constraint compared to objects of similar mass and ages in young moving groups for which the radius is constrained through bolometric properties (Filippazzo et al. 2015).

Young BDs have a significant self-luminosity, and a young system would be significantly easier to detect as a double-lined spectroscopic binary. Furthermore, the TOI-1278 system could consist of an unresolved binary system with the transiting companion around one of the two components. While there are

\footnotetext{
32 See also http://www.pas.rochester.edu/ emamajek/EEM_dwarf_UBVIJHK_ colors_Teff.txt.
}
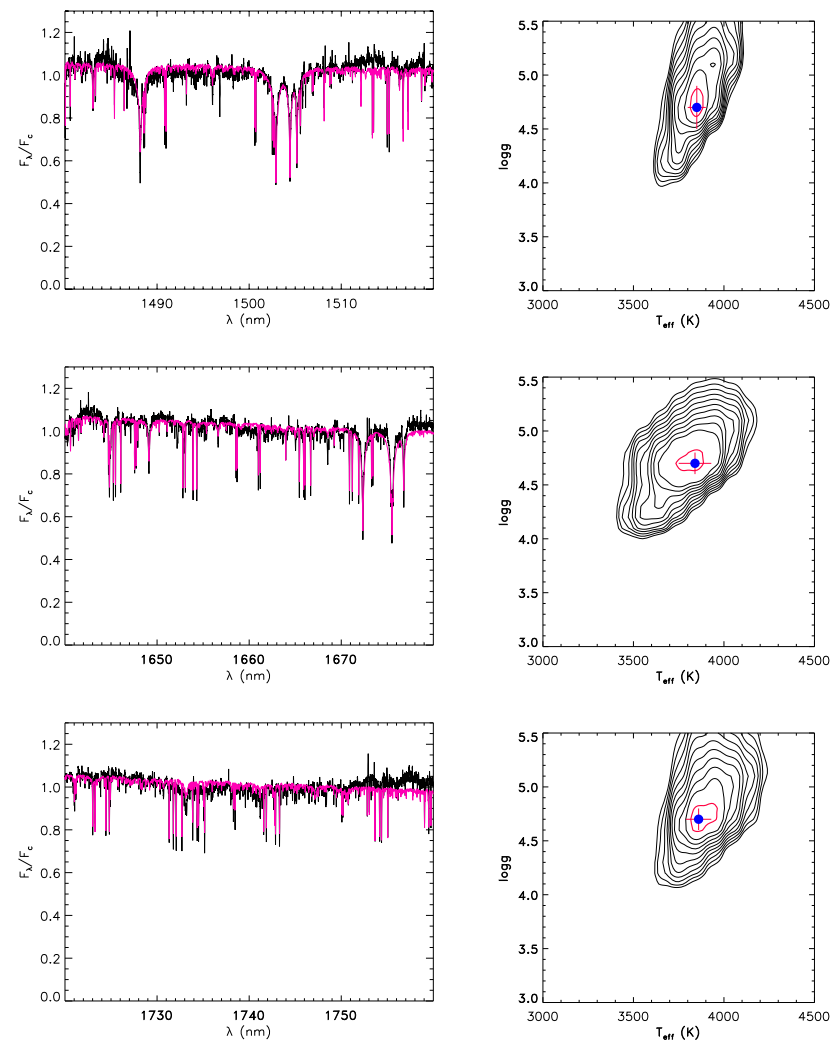

Figure 6. Left panels: continuum-normalized co-added SPIRou spectrum of TOI-1278 in three regions (black full lines) with the best-fitting synthetic spectrum over-plotted (red dotted lines). Right panels: $\chi^{2}$ contour maps in the $T_{\text {eff }}-\log g$ plane. In each panel, the $1 \sigma$ confidence level is denoted by the red contour. The best values and error bars on $T_{\text {eff }}$ and $\log g$ are also indicated.

a number of well-established tools to rule out a number of scenarios for transiting companions (see Sections 2.1 and 2.4), simple color-magnitude diagram analysis can already exclude a number of configurations.

\subsection{Stellar Parameters from SPIRou Spectra}

The determination of the atmospheric parameters $\left(T_{\text {eff }}\right.$ and $\log g$ ) and projected rotational velocity $(v \sin i)$ was accomplished through the code ROTFIT (e.g., Frasca et al. 2017).

We adopted a grid of synthetic BT-Settl spectra as templates (Allard et al. 2010) at $[\mathrm{Fe} / \mathrm{H}]=-0.5,0.0$, and 0.3 , and effective temperature in the range $3000-4500 \mathrm{~K}$ (in steps of $100 \mathrm{~K}$ ) and $\log g$ from 5.5 to $2.5 \mathrm{dex}$ (in steps of $0.5 \mathrm{dex}$ ).

ROTFIT finds the best values of atmospheric parameters and $v \sin i$ by minimizing the $\chi^{2}$ of the difference between the observed and synthetic spectra in specific spectral segments. We used the $H$ band (eight $40 \mathrm{~nm}$ wide segments) as well as regions centered on strong lines within $J$ and $K(1290-1330 \mathrm{~nm}$ and 1960-2000 nm) for the ROTFIT analysis. These domains avoid the stronger telluric absorption bands between photometric bandpasses that may display significant correction residuals, and avoids the increased thermal noise on the red side of the $K$ band. Some regions within $Y$ and $J$ are relatively clean of tellurics, but have fewer strong and sharp spectroscopic features than the regions selected for the analysis; this manifests itself in the very poor RV content of the $Y$ and $J$ bands for M dwarfs (e.g., Figueira et al. 2016; Artigau et al. 2018b). Figure 6 shows sample regions for the fit on the 
telluric-corrected co-added SPIRou spectrum. Both templates and observed spectra are normalized to the local continuum, and the templates are degraded to the SPIRou spectral resolution and resampled on the points of the target spectrum. The templates are also artificially broadened by convolution with a rotational profile whose $v \sin i$ spans a range of values to find the minimum of $\chi^{2}$. For each spectral segment, the best values of $T_{\text {eff }}$ and $\log g$ are found by interpolation in the grid of templates, as shown in the right panels of Figure 6.

The values of $T_{\text {eff }}=3799 \pm 42 \mathrm{~K}, \log g=4.68 \pm 0.10$ dex, and $v \sin i=1.10 \pm 0.86 \mathrm{~km} \mathrm{~s}^{-1}$ are the weighted means of the values from each $i$ th spectral region, where the weights are $w_{i}=1 / \sigma_{i}^{2}\left(\sigma_{i}\right.$ is the error of the parameter for the $i$ th segment. The analysis was performed at three metallicity values, and the lowest $\chi^{2}$ value was obtained for $[\mathrm{Fe} / \mathrm{H}]=0.0$. The uncertainty of each parameter is the largest between the standard error of the weighted mean and the weighted standard deviation. The value of $v \sin i$ is significantly smaller than the spectral resolution of SPIRou $\left(\sim 4.3 \mathrm{~km} \mathrm{~s}^{-1}\right)$, and is sensitive to other broadening effects, such as the macro-turbulence, that are not distinguishable from rotation at low $v \sin i$ values. The $v \sin i$ should therefore be taken as a very approximate value.

\subsection{Refining Metallicity Measurements}

The high-resolution spectra of TOI-1278 let us determine the metallicity of the star with comprehensive examinations of individual Fe I lines in all $Y, J_{2 M}, H_{2 M}$, and $K_{2 M}$ bands. BTSettl and Astrophysical Chemical Equilibrium Solver (ACES) models (Allard et al. 2011; Husser et al. 2013) are available at varying resolution over the SPIRou domain. The sampling of BT-Settl models is not uniform; it is typically $0.20 \AA$, but it can be as low as $0.05 \AA$, which is comparable with that of SPIRou, inside strong spectral lines. The determination of $T_{\text {eff }}$ and $\log g$ discussed in the section above is not strongly sensitive to the resolution, but in contrast, metallicity measurements are more strongly sensitive to the data resolution. We therefore decided to use PHOENIX ACES models, available at a resolution higher than that of SPIRou, for metallicity measurements of all identified individual Fe I absorption lines. To do this, we generated multiple synthetic spectra for the fixed effective temperature and $\log g$ of $3800 \mathrm{~K}$ and 4.5 dex, respectively, and the metallicity range of -1.5 to $0.5 \mathrm{dex}$. The $T_{\text {eff }}$ and $\log g$ values were adopted from ROTFIT results (see Table 2) and then rounded to the nearest $100 \mathrm{~K}$ and 0.5 dex.

To match the original resolution and sampling of the ACES models to that of the observed data, we degraded and convolved all of the generated synthetic data with respect to a SPIRou spectrum. Then we used the Vienna Atomic Line Database (VALD; Ryabchikova et al. 2015; Kupka et al. 2000; Piskunov et al. 1995) to identify all visible Fe I lines on the models. Finally, we performed $\chi^{2}$ analyses between the data and the models on each line individually. Note that in total, 78 $\mathrm{Fe}$ I lines were detected and used in our analysis. The result of this chemical spectroscopy is a metallicity of $[\mathrm{Fe} / \mathrm{H}]=$ $-0.01 \pm 0.28$, where the conservative uncertainty is directly from the standard deviation of all of the measurements. Hence we conclude that TOI-1278 has a solar metallicity, in agreement with the ROTFIT results.
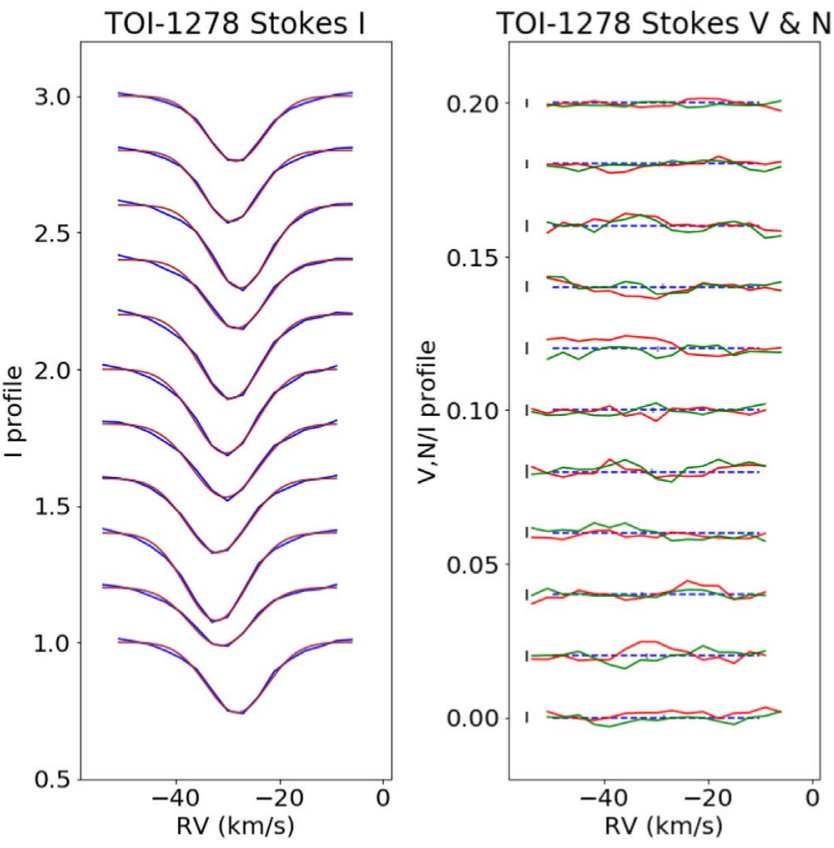

Figure 7. Stokes I (left) and Stokes V (right) of all polarization sequences obtained on TOI-1278. The error bars close to Stokes V profiles show the mean uncertainty on each data point. The green curves on the right plot show the null check profiles. Superimposed to the intensity profiles is a Gaussian fit.

\subsection{Spectropolarimetry with SPIRou}

Given that each visit to TOI-1278 is composed of a polarimetric sequence, it is possible to combine the individual spectra of each beam to have access to the circular polarization of the stellar surface (Donati et al. 1997, 2020). Using leastsquare deconvolution and a line list, a mean profile is obtained for the intensity spectrum (Stokes I profile) as well as for the polarized spectrum (Stokes V profile). The line list used for TOI-1278 is based on the VALD database for an effective temperature of $3750 \mathrm{~K}$ and minimum line depth of 0.15 of the continuum; it contains 500 atomic lines. The description of the data analysis method as included in the APERO pipeline is given in Martioli et al. (2020). Figure 7 shows the resulting Stokes I and Stokes V profiles for the whole data set. The noise level in the Stokes V profile corresponds to a few $10^{-4}$ of the intensity spectrum, with no significant detection in the available time series. Using the relationship between the mean Landé factor, the mean wavelength, and the Stokes V and I profiles as introduced in Donati et al. (1997), one can find a $1 \sigma$ upper limit of 22 to 35 Gauss for the longitudinal field on these visits.

\section{Data Analysis and Results}

To constrain the orbital and physical parameters of TOI$1278 \mathrm{~B}$, we performed a joint analysis of the SPIRou RV data along with the TESS and ground-based transits. We used the Python package exoplanet (Foreman-Mackey et al. 2020), which computes analytical transit models using starry (Luger et al. 2019) and solves the Kepler equation with a built-in solver. The exoplanet framework uses PyMC3 (Salvatier et al. 2016) to generate Bayesian models and perform gradient-based Markov Chain Monte Carlo. Models in PyMC3 are built with the Theano (Al-Rfou et al. 2016) numerical infrastructure. 
With this framework, we modeled the four transits (two from TESS, one from WBRO, and one from CAO) and the full SPIRou RV time series simultaneously. We included a separate baseline flux $f_{0}$ and quadratic limb-darkening coefficients $\left\{u_{1}, u_{2}\right\}$ for each instrument. For TESS, the coefficients are from Tables 2 and 5 of Claret (2018) for Teff and logg values respectively, and are held fixed to $\{0.2336,0.3710\}$. For CAO, we used EXOFAST (Eastman et al. 2013) to interpolate tables from Claret \& Bloemen (2011). ${ }^{33} \mathrm{We}$ assumed $[\mathrm{Fe} / \mathrm{H}]=0$ (see Section 3.3) and fixed the resulting $I_{\mathrm{c}}$ bandpass coefficients to $\{0.2776,0.3528\}$. Since the WBRO transit was observed without any filter, we chose to let $\left\{u_{1}, u_{2}\right\}$ vary following a Gaussian distribution $\mathcal{N}(0.3,0.1)$.

We used relatively narrow uniform priors on the period $P$ and the time of mid-transit $T_{0}$, but their ranges are still several orders of magnitude larger than the uncertainties from the preliminary analysis by the TESS team (DV: $P=14.4762 \pm$ 0.0022 days, $T_{0}=2458711.959717 \pm 0.0015$ BJD). We also imposed Gaussian priors on the stellar radius $R_{\mathrm{s}}$ and mass $M_{\mathrm{s}}$ using values from Table 2 . We used uniform priors on the orbital eccentricity $e$ and argument of periastron $\omega$, as well as for the systemic velocity $\gamma$. For the RV semi-amplitude $K$, we used a broad log-uniform prior to sample several orders of magnitude uniformly. The transit impact parameter $b$ has a uniform prior between 0 and $1+R_{\mathrm{p}} / R_{\mathrm{s}}$. Since the transit is grazing, a strong degeneracy exists between $b$ and the planetary radius $R_{\mathrm{p}}$. To alleviate this degeneracy and assess its importance, we compared three different priors on $R_{\mathrm{p}}$ : a broad uniform prior between 0.0 and $2.5 R_{\mathrm{Jup}}$, a Gaussian prior based on the latest evolutionary models described in Section 5.3, and a Gaussian prior derived from the confirmed exoplanet population. To construct the latter, we follow an approach similar to that of Bayliss et al. (2018). We used the Pythonbased tool masterfile, ${ }^{34}$ which relies on data from the NASA Exoplanet Archive ${ }^{35}$ to obtain the population of transiting exoplanets with $M_{\mathrm{p}}>0.5 M_{\text {Jup }}$ and with an incident flux smaller than 50 times that of the Earth, yielding a sample of 21 planets. We then used the distribution of their radii to derive a Gaussian prior with a mean of $1.010 R_{\text {Jup }}$ and a standard deviation of $0.331 R_{\text {Jup }}$.

We sampled the joint posterior distribution of the 15 model parameters, $\left\{P, T_{0}, e, \omega, K, R_{\mathrm{p}}, b, M_{\mathrm{s}}, R_{\mathrm{s}}, \gamma, f_{0, \mathrm{TESS}}, f_{0, \mathrm{WBRO}}\right.$, $f_{0, \mathrm{CAO}}, u_{1, \mathrm{WBRO}}$, and $\left.u_{2, \mathrm{WBRO}}\right\}$, with the No-U-Turn Sampler (NUTS) from PyMC3. We ran four chains with 4000 tuning steps and 4000 draws. The planetary radius posterior distribution for each of the three priors investigated is shown in Figure 8. All priors yield a posterior value for $R_{\mathrm{p}}$ close to $1 R_{\mathrm{Jup}}$, but the width of these distributions varies significantly depending on the strength of the prior. Parameters other than $R_{\mathrm{p}}$ and $b$ were not significantly affected by this change of prior. For this reason, in the following, we only report posterior distributions on parameters that used the "Demographics" prior on $R_{\mathrm{p}}$. This prior provides an insight on reasonable $R_{\mathrm{p}}$ values without completely excluding the possibility of an inflated radius due to irradiation.

For each parameter, Table 3 reports the final prior distribution used and the median posterior value, along with uncertainties corresponding to the 16th and 84th percentiles. Figure 1 shows the resulting transit model on each data set. In

\footnotetext{
33 See also http://astroutils.astronomy.ohio-state.edu/exofast/limbdark.shtml.

34 https://github.com/AntoineDarveau/masterfile

35 https://exoplanetarchive.ipac.caltech.edu
}

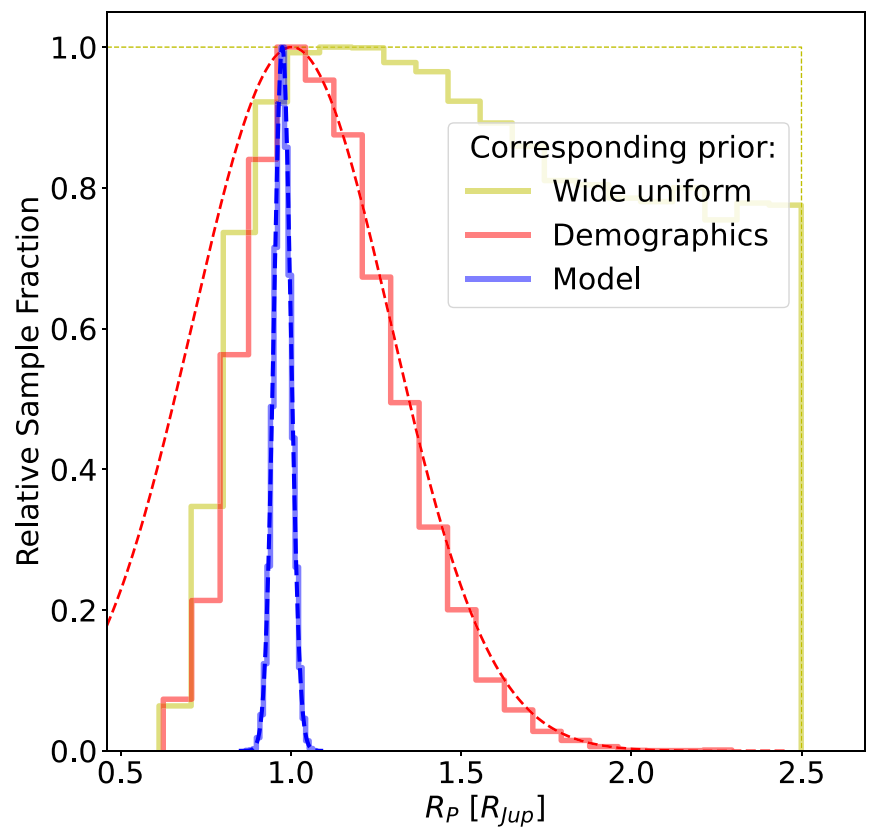

Figure 8. Planetary radius posterior distribution for three different priors. The solid lines show the histogram of the posterior distributions, and the corresponding prior probability density functions are shown as dashed lines of the same color. The prior from evolutionary models (blue), as described in Section 5.3, is $\mathcal{N}(0.975,0.025)$ and yields $R_{\mathrm{p}}=0.976 \pm 0.025 R_{\text {Jup }}$. The prior derived from the confirmed exoplanet population (red) is $\mathcal{N}(1.010,0.331)$ and yields $R_{\mathrm{p}}=1.09_{-0.20}^{+0.24} \quad R_{\text {Jup. }}$. The broad uniform prior $\mathcal{U}(0.0,2.5)$ (yellow) yields $R_{\mathrm{p}}=1.56_{-0.53}^{+0.63} R_{\text {Jup. }}$

Figure 3, the full RV time series and the phase-folded RVs are both shown along with the best-fit model and the residuals. The joint posterior distribution is shown in Figure 9.

Along with a planetary mass $M_{p}=18.5 \pm 0.5 M_{\mathrm{Jup}}$, a radius of $1.09_{-0.20}^{+0.24} R_{\text {Jup }}$ yields a density $\rho_{p}=18_{-8}^{+14} \mathrm{~g} \mathrm{~cm}^{-3}$. We find a slightly eccentric orbit $(e=0.013 \pm 0.004)$. We also test a circular orbit model with $e=0$ and $\omega=0$ following the same modeling procedure as above. All parameters in this circular model are consistent with the values obtained with the eccentric model. However, the eccentric model is strongly favored by the Bayesian information criterion (BIC), with $\triangle \mathrm{BIC}=9$. Using the eccentric model, we constrain the time of secondary eclipse to be $T_{\mathrm{sec}}=1719.143 \pm 0.015$ (BJD—2 457000$)$. This corresponds to an orbital phase of $0.496 \pm 0.001$. Orbital evolution and the circularization timescale are discussed in Section 5.2.

We also performed a sequential analysis where the transits were modeled first, and the resulting $P$ and $T_{0}$ posterior distributions were used as priors in the RV Keplerian model. This approach has the disadvantage of not accounting for possible impacts of the eccentricity (which is better constrained by the RV data in this case) on the transit model. However, we note that results from both approaches are in excellent agreement, yielding similar posterior distributions with median values consistent within $1 \sigma$. This is notable as the sequential analysis does not rely on the stellar parameters as inputs.

Combining the proper motion and parallax from Gaia eDR3 with the systemic RV measurement $(\gamma=-29.334 \pm$ $0.007 \mathrm{~km} \mathrm{~s}^{-1}$ ) derived in this section makes it possible to determine the $U V W$ space velocities of TOI-1278 with an unprecedented precision (the Gaia eDR3 RV for TOI-1278 is $\left.-29.6 \pm 1.1 \mathrm{~km} \mathrm{~s}^{-1}\right)$. However, doing so requires correcting 


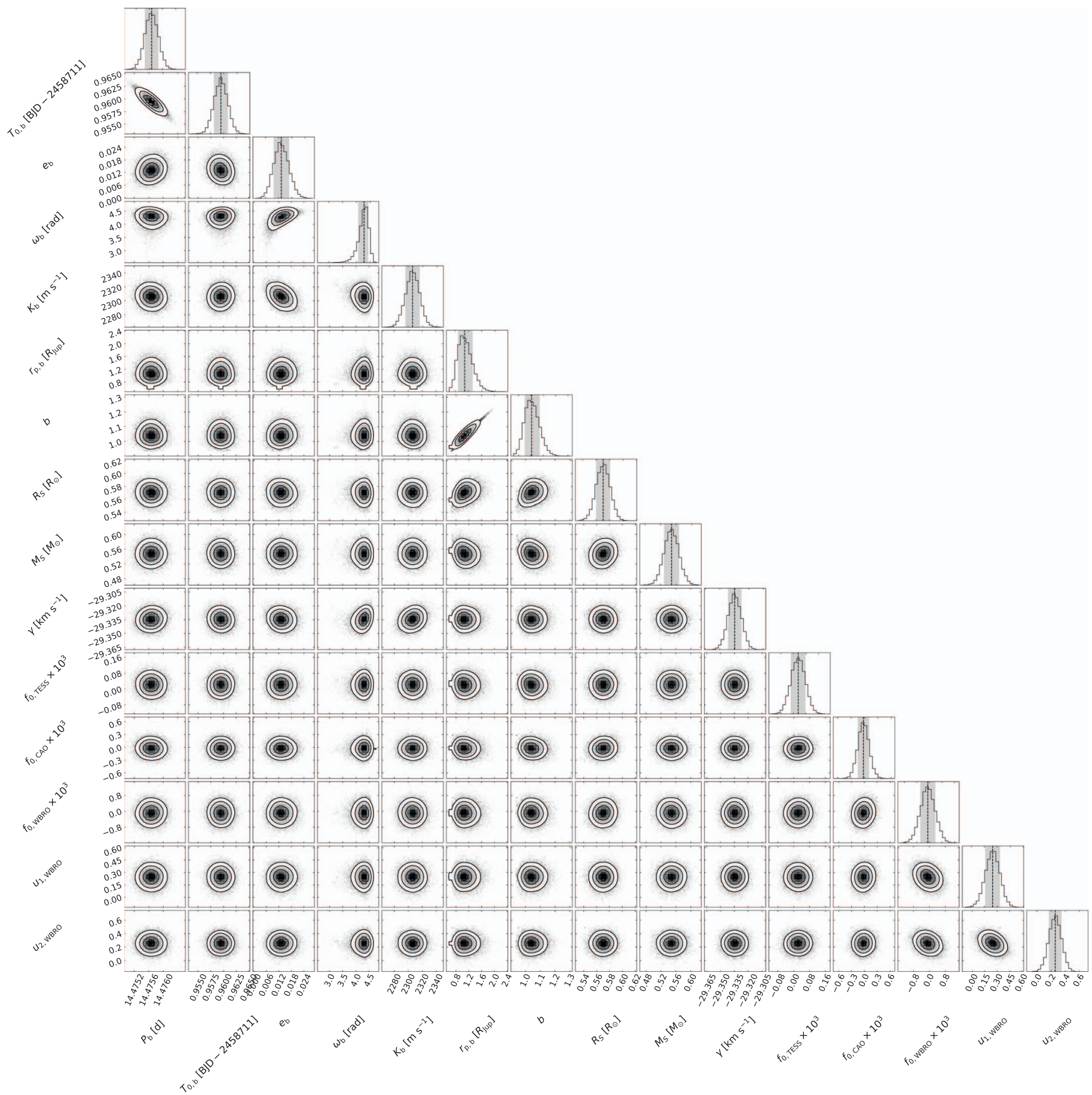

Figure 9. Posterior distribution of the joint RV and transit model parameters. The dashed lines and gray shaded areas show the median and the $1 \sigma$ interval for each parameter.

two systematic effects that become important with meter-perlevel instrumental precision: gravitational reddening (Einstein 1917) and convective blueshift (e.g., Meunier et al. 2017). Our mass and radius estimations allow us to calculate the expected gravitational redshift of $0.63 \pm 0.03 \mathrm{~km} \mathrm{~s}^{-1}$. Estimating the effect of convective blueshift with a high accuracy is much harder, as empirical data on that subject is sparse, especially for M dwarfs. Löhner-Böttcher et al. (2019) estimated that this effect blueshifts the perceived RV of the Sun by about $0.3 \pm 0.2 \mathrm{~km} \mathrm{~s}^{-1}$. Only one recent study by Baroch et al. (2020) investigated this effect for $\mathrm{YZCm}$, a binary M4 dwarf, and found that it ranges from +0.007 to $+0.247 \mathrm{~km} \mathrm{~s}^{-1}$, corresponding to a very slight redshift rather than a blueshift, which is expected for most stars. (Dai et al. 2018) investigated this effect using a larger sample from Gaia DR2, and found an empirical blueshift of $-0.2 \pm 0.2 \mathrm{~km} \mathrm{~s}^{-1}$ for early-K dwarfs. We therefore estimate that the convective blueshift for a field-aged M0 star is likely of about $0.0 \pm 0.2 \mathrm{~km} \mathrm{~s}^{-1}$, as a middle case between those of YZCMi and early-K dwarfs. The total redshift to which TOI-1278 is subjected is consequently $0.6 \pm 0.2 \mathrm{~km} \mathrm{~s}^{-1}$. We therefore estimate a heliocentric RV of $-29.9 \pm 0.2 \mathrm{~km} \mathrm{~s}^{-1}$ for TOI-1278, and we use this value to 
Table 1

Basic Data of TOI-1278

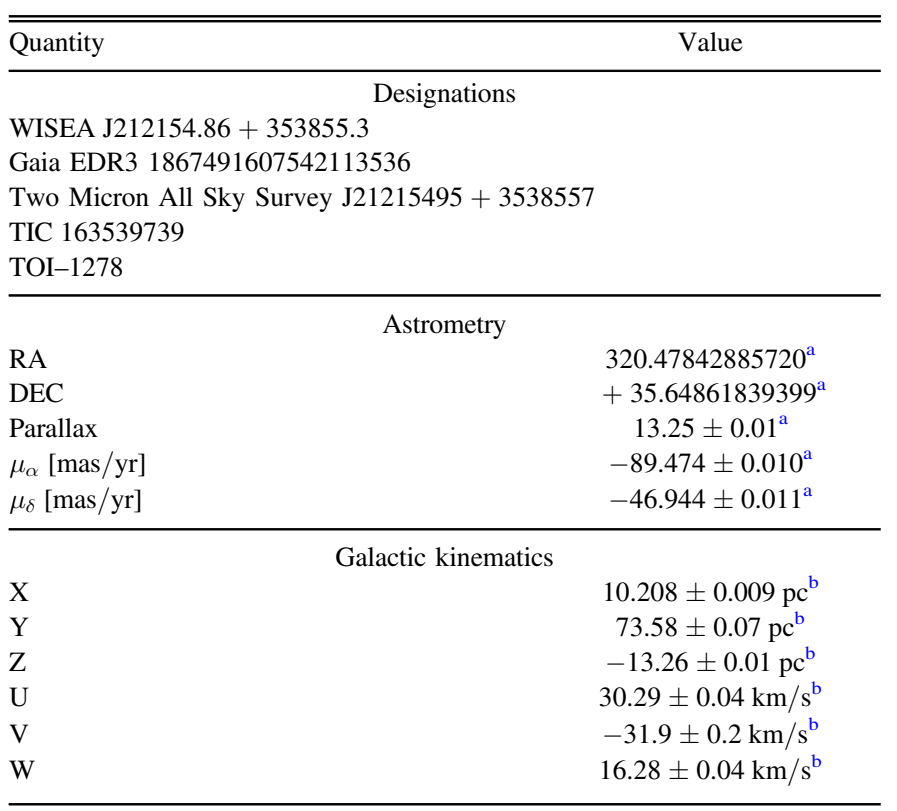

Notes.

${ }^{a}$ Gaia EDR3 (Gaia Collaboration et al. 2021).

b This work, combining GAIA astrometry and SPIRou velocimetry.

calculate the space velocities listed in Table 1. Overall, the space velocities of TOI-1278 are located just within the $2 \sigma$ velocity dispersion of the Galactic thin disk of Bensby et al. (2003), indicating that TOI-1278 is likely not much older than $\approx 8 \mathrm{Gyr}$.

\section{Discussion}

\subsection{On the Paucity of Close-in BD Companions to M Dwarfs}

The number of known BD companions in close-in orbits around main-sequence stars is relatively small (see full compilation in Table 4 of Mireles et al. 2020), although they are easier to find in general than planetary companions. This is expected from a formation point of view, with close-in binaries having mass ratios tending toward unity (Bate 2000). While most such companions orbit sunlike stars, TOI-1278 combines relatively rare properties; there are few close-in $(<0.1 \mathrm{au})$ companions to $\mathbf{M}$ dwarfs that are more massive than Saturn (see Figure 10 and Table 4 for a compilation of 0.5-78 $M_{\text {Jup }}$ companions to $\mathrm{M}$ dwarfs).

An order-of-magnitude upper limit on the abundance of TOI1278-like systems can be determined from the fact that RV surveys of the solar neighborhood have failed to uncovered similar systems, even though it would have been well above their detection thresholds. The High Accuracy Radial velocity Planet Searcher survey of southern M dwarfs (Bonfils et al. 2013) surveyed 102 stars; the most massive planet in the sample (G1876 b; Delfosse et al. 1998) has a mass of $2.64 M_{\text {Jup }}, \sim 7$ times lighter than TOI-1278 B. The CARMENES search for exoplanets around M dwarfs (Reiners et al. 2018) surveyed 324 nearby stars (with some overlap between samples) and did not report any such massive companions. Overall, one can safely say that the occurrence of such systems is well below $1 \%$.

NGTS-1 $b$ is the most massive transiting planetary companion to an M dwarf (Bayliss et al. 2018). It is $\sim 20$ times less massive $\left(0.8 M_{\text {Jup }}\right)$ and orbits on a shorter 2.647 day period. There are a number of comparable (10-15 $\left.M_{\text {Jup }}\right)$ companions to $\mathrm{M}$ dwarfs on distant orbits uncovered through direct imaging (e.g., GU Psc b, 2M J2126-81 b, CHXR 73 b, DH Tau b, 2M 0103-55(AB)b; Naud et al. 2014; Luhman et al. 2006; Deacon et al. 2016; Itoh et al. 2005; Delorme et al. 2013). These companions form well outside the extent of protoplanetary disks around $\mathrm{M}$ dwarfs, and the statistical properties in terms of orbital separation and mass suggest a different formation mechanism than for close-in planetary companions (Baron et al. 2019). The mass ratio of the TOI-1278 system $(\sim 31)$ cannot be explained through a process involving a planetary disk; the mass involved is larger than the total gas mass in planetary disks, which has a plateau around $10 M_{\text {Jup }}$ (Bergin \& Williams 2018). As M-dwarf luminosity falls rapidly with decreasing mass $\left(L_{\star} \propto M_{\star}^{3}\right)$, uncovering companions with small $q$ values is challenging due to the contrast ratio involved, and there are significant biases against the discovery of low- $q$ binaries. Detecting TOI-1278-like systems requires RV followups, as gigayear-old $1 M_{\text {Jup }}$ to $0.1 M_{\odot}$ companions all have radii in the $0.8-1.0 R_{\text {Jup }}$; transit alone does not set a significant constraint on the nature of the companion. Conversely, the RV signal of such a companion will be huge, from $100 \mathrm{~m} \mathrm{~s}^{-1}$ to many kilometers per second, implying that the companions to faint host stars can be monitored. The paucity of 10-30 $M_{\text {Jup }}$ may also be arising from a bias in the follow-up strategy by community members; with an impact parameter slightly lower, TOI-1278 B's transit would have a relative depth of $\sim 4 \%$ and be flagged as a likely eclipsing binary. A dedicated search for similar objects within the TESS data set complemented with a follow-up of a statistically representative set of objects will be needed to assess the abundance of objects similar to TOI1278 B. Current follow-ups, focusing on smaller-radius planets around relatively bright and nearby $M$ dwarfs and avoiding possible eclipsing binaries, are naturally biased against the discovery of objects similar to TOI-1278 B. The true abundance of such systems therefore remains to be established.

Among transiting companions to $\mathrm{M}$ dwarfs, only a handful are in the BD mass regime. The LHS 6343 system is composed of a visual binary of two mid-Ms, (0."55 apart; 0.358 and $0.292 M_{\odot}$ ), one component orbited by a close-in BD (Johnson et al. 2011). LHS $6343 \mathrm{C}$ orbits on a 12.71 day orbit around LHS $6343 \mathrm{~A}$, and has a kinematically derived mass of $62.7 \pm 2.4 M_{\text {Jup }}$. It has a mass ratio of only $\sim 6$ with its host star. The LP 261-75 b system (Irwin et al. 2018) consists of a much tighter orbit ( $\sim 1.8$ day orbit) by a BD close to the stellar/ substellar limit at $65 M_{\mathrm{Jup}}$, and a mass ratio of $\sim 4.6$.

\subsection{Eccentricity and Circularization Timescale}

As discussed in Section 4, we find that a slightly eccentric orbit $(e=0.013 \pm 0.004)$ is preferred over a circular one. We compute the expected timescale for tidal orbital circularization of TOI-1278 B by using Equation (25) of Goldreich \& Soter (1966) as rewritten by Patra et al. (2017):

$$
\tau_{e}=\frac{e}{d e / d t}=\frac{2 Q_{p}}{63 \pi} \frac{M_{p}}{M_{\star}}\left(\frac{a}{R_{p}}\right)^{5} P_{\mathrm{orb}} .
$$

$Q_{p}$ is the tidal dissipation parameter of the companion, depending on its internal structure. We assume here that $Q_{p}=10^{3.5}$, as determined by Heller et al. (2010) for the BD. With a value of $16 \times 10^{9} \mathrm{yr}$, the timescale $\tau_{e}$ is comparable to 
Table 2

Bulk Properties of TOI-1278

\begin{tabular}{|c|c|}
\hline Quantity & Value \\
\hline \multicolumn{2}{|c|}{ Stellar parameters } \\
\hline Mass $\left(M_{\odot}\right)$ & $0.55 \pm 0.02^{\mathrm{a}}$ \\
\hline Radius $\left(R_{\odot}\right)$ & $0.573 \pm 0.012^{\mathrm{b}}$ \\
\hline $\log g$ & $4.68 \pm 0.10^{\mathrm{b}}$ \\
\hline Luminosity $\left(\mathrm{L}_{\odot}\right)$ & $0.0614 \pm 0.0001^{\mathrm{b}}$ \\
\hline$T_{\text {eff }}(\mathrm{K})$ & $3799 \pm 42^{b}$ \\
\hline$v \sin i\left(\mathrm{~km} \mathrm{~s}^{-1}\right)$ & $1.10 \pm 0.86^{\mathrm{b}}$ \\
\hline $\mathrm{A}_{V}(\mathrm{mag})$ & $-0.01 \pm 0.10^{\mathrm{b}}$ \\
\hline$[\mathrm{Fe} / \mathrm{H}]$ & $-0.01 \pm 0.28^{\mathrm{b}}$ \\
\hline Spectral type & $\mathrm{M} 0 \mathrm{~V}^{\mathrm{b}}$ \\
\hline \multicolumn{2}{|c|}{ Photometry } \\
\hline$B$ & $15.07 \pm 0.05^{\mathrm{b}}$ \\
\hline$V$ & $13.51 \pm 0.03^{\mathrm{b}}$ \\
\hline$R_{c}$ & $12.60 \pm 0.04^{\mathrm{b}}$ \\
\hline$I_{c}$ & $11.70 \pm 0.04^{\mathrm{b}}$ \\
\hline$G$ & $12.740 \pm 0.003^{c}$ \\
\hline$g$ & $14.078 \pm 0.007^{\mathrm{d}}$ \\
\hline$r$ & $12.898 \pm 0.001^{\mathrm{d}}$ \\
\hline$i$ & $12.30 \pm 0.04^{\mathrm{d}}$ \\
\hline$z$ & $12.5 \pm 0.4^{\mathrm{d}}$ \\
\hline$y$ & $12.07 \pm 0.05^{\mathrm{d}}$ \\
\hline$J_{2 M}$ & $10.601 \pm 0.025^{\mathrm{e}}$ \\
\hline$H_{2 M}$ & $9.939 \pm 0.021^{\mathrm{e}}$ \\
\hline$K_{\mathrm{s}, 2 \mathrm{M}}$ & $9.735 \pm 0.012^{\mathrm{e}}$ \\
\hline$W 1$ & $9.629 \pm 0.022^{f}$ \\
\hline$W 2$ & $9.591 \pm 0.020^{f}$ \\
\hline$W 3$ & $9.443 \pm 0.03^{\mathrm{f}}$ \\
\hline
\end{tabular}

Notes.

a Stassun et al. (2018).

b This work.

c Gaia EDR3 (Gaia Collaboration et al. 2021).

d Pan-STARRS1 (Flewelling et al. 2020).

e Two Micron All Sky Survey (Skrutskie et al. 2006).

${ }^{f}$ AllWISE (Kirkpatrick et al. 2014).

the age of the Universe, suggesting that the orbit has not had time to fully circularize. This further implies that an eccentric orbit does not require the presence of an external exciting companion to be explained. We note that $\tau_{e}$ scales as the fifth power of $R_{\mathrm{p}}$, and that this radius is currently only constrained by models for $\mathrm{M}$ dwarfs and BDs.

\subsection{Model-derived Properties and Characterization Prospects}

BDs are ever cooling objects that gradually lose the heat from their initial contraction. In the absence of a luminosity measurement, only the combination of age and mass provides some constraint on the BD temperature. While the mass of TOI-1278 B is well constrained at $18.5 \pm 0.5 M_{\text {Jup }}$, its age is not. Its $\gtrsim 100 \mathrm{Myr}$ lower limit is set by the uninflated radius of the parent star, while the thin-disk kinematics sets a limit at $\lesssim 9$ Gyr (del Peloso et al. 2005). Considering a formation rate that is uniform through time in the galactic disk, it is unlikely to be at the lower end of this range when the temperature and radius rapidly decrease. Assuming a uniform prior distribution in age for the galactic disk, the corresponding $1 \sigma$ confidence level interval for the age of TOI-1278 is therefore 1.4-7.6 Gyr (14th and 86th percentiles of the distribution).
Table 3

Model Parameters for the TOI-1278 System

\begin{tabular}{|c|c|c|}
\hline Quantity & Prior & Posterior \\
\hline$P$ [day $]$ & $\mathcal{U}(13.476,15.476)$ & $14.47567 \pm 0.00021$ \\
\hline$T_{0}[\mathrm{BJD}-2457000]$ & $\mathcal{U}(1711,1713)$ & $1711.9595 \pm 0.0013$ \\
\hline$R_{\mathrm{p}}\left[R_{\mathrm{Jup}}\right]$ & $\mathcal{N}(1.010,0.331)$ & $1.09_{-0.20}^{+0.24}$ \\
\hline$b$ & $\mathcal{U}\left(0,1+R_{\mathrm{p}} / R_{S}\right)$ & $1.04_{-0.05}^{+0.06}$ \\
\hline$M_{\mathrm{S}}\left[M_{\odot}\right]$ & $\mathcal{N}(0.55,0.02)$ & $0.54 \pm 0.02$ \\
\hline$R_{\mathrm{s}}\left[R_{\odot}\right]$ & $\mathcal{N}(0.573,0.012)$ & $0.57 \pm 0.01$ \\
\hline$e$ & $\mathcal{U}(0,1)$ & $0.013 \pm 0.004$ \\
\hline$\omega[\mathrm{rad}]$ & $\mathcal{U}(0,2 \pi)$ & $4.29_{-0.21}^{+0.15}$ \\
\hline$K\left[\mathrm{~m} \mathrm{~s}^{-1}\right]$ & $\log U\left(10^{-4}, 10^{4}\right)$ & $2306 \pm 10$ \\
\hline$\phi_{\mathrm{sec}}$ & - & $0.496 \pm 0.001$ \\
\hline$M_{\mathrm{p}}\left[M_{\mathrm{Jup}}\right]$ & - & $18.5 \pm 0.5$ \\
\hline$i[\mathrm{deg}]$ & - & $88.3 \pm 0.1$ \\
\hline$a[\mathrm{au}]$ & - & $0.095 \pm 0.001$ \\
\hline$\rho_{\mathrm{p}}\left[\mathrm{g} \mathrm{cm}^{-3}\right]$ & - & $18_{-8}^{+14}$ \\
\hline$\gamma\left[\mathrm{k} \mathrm{m} \mathrm{s}^{-1}\right]$ & $\mathcal{U}(-35,-25)$ & $-29.334 \pm 0.007$ \\
\hline$f_{0, \mathrm{TESS}} \times 10^{3}$ & $\mathcal{N}(0,10000)$ & $0.02 \pm 0.04$ \\
\hline$f_{0, \text { WBRo }} \times 10^{3}$ & $\mathcal{N}(0,10000)$ & $-0.08 \pm 0.46$ \\
\hline$f_{0, \mathrm{CAO}} \times 10^{3}$ & $\mathcal{N}(0,10000)$ & $-0.02 \pm 0.12$ \\
\hline$u_{1, \mathrm{WBRO}}$ & $\mathcal{N}(0.3,0.1)$ & $0.25 \pm 0.09$ \\
\hline$u_{2, \mathrm{WBRO}}$ & $\mathcal{N}(0.3,0.1)$ & $0.26 \pm 0.09$ \\
\hline
\end{tabular}

We used the latest evolutionary models for cool $\mathrm{T}$ and $\mathrm{Y}$ dwarfs (Phillips et al. 2020) to determine the range of properties expected for TOI-1278 B. Figure 11 shows the radius, temperature and luminosity evolution with age for the range of masses plausible for TOI-1278. Constraints on the age of the system translate into constraints on these three parameters. These constraints on the age of the system point toward a cool-down temperature ranging from $600 \mathrm{~K}$ to $380 \mathrm{~K}$ ( $1 \sigma$ confidence), but as high as $1360 \mathrm{~K}$ for the lower end of the age range; these values correspond to a bulk luminosity ranging from $10^{-4.4}$ to $10^{-6.8} L_{\odot}$, to be compared to the host-star luminosity of $5.9 \times 10^{-2} L_{\odot}$. Expressed in terms of BD spectral sequence, TOI-1278 B is therefore expected to have a spectral type of L7-L8 if we assume an age of $100 \mathrm{Myr}$, T8-T9 for an age of 1.5 Gyr or Y1 for an age of 7.6 Gyr (Faherty et al. 2016; Kirkpatrick et al. 2019).

Assuming the $1 \sigma$ confidence interval for the age, the radius of TOI-1278 B is remarkably well constrained at $0.975 \pm 0.025 R_{\text {Jup }}$; degeneracy pressure dominates the radius evolution of objects in this age and mass range, implying that the object's radius is (nearly) constant through time. This provides an important prior for transit light-curve fitting; the $b \sim 1$ impact parameter implies that the light curve alone sets little constraint on the planetary radius, with a strong degeneracy between $b$ an $R_{\mathrm{p}}$ as seen from the diagonal in the corresponding panel of Figure 9. A much larger planet (up to $R_{\mathrm{p}} \sim 2 R_{\mathrm{Jup}}$ ) with an impact parameter of $b \sim 1.2$ would be allowed from transit light-curve analysis alone, but is rejected by evolutionary models.

One important output of evolutionary models is the per-band flux, which can be combined with the host-star photometry (see Table 5) to derive a flux ratio. While the system is far too close to be directly resolved with instrumentation in the foreseeable future ( $\sim 1$ mas at elongation), it is more likely to be detected through cross-correlation techniques or eclipse photometry or spectroscopy. In the most optimistic case, the contrast ratio 
TOI-1278 system

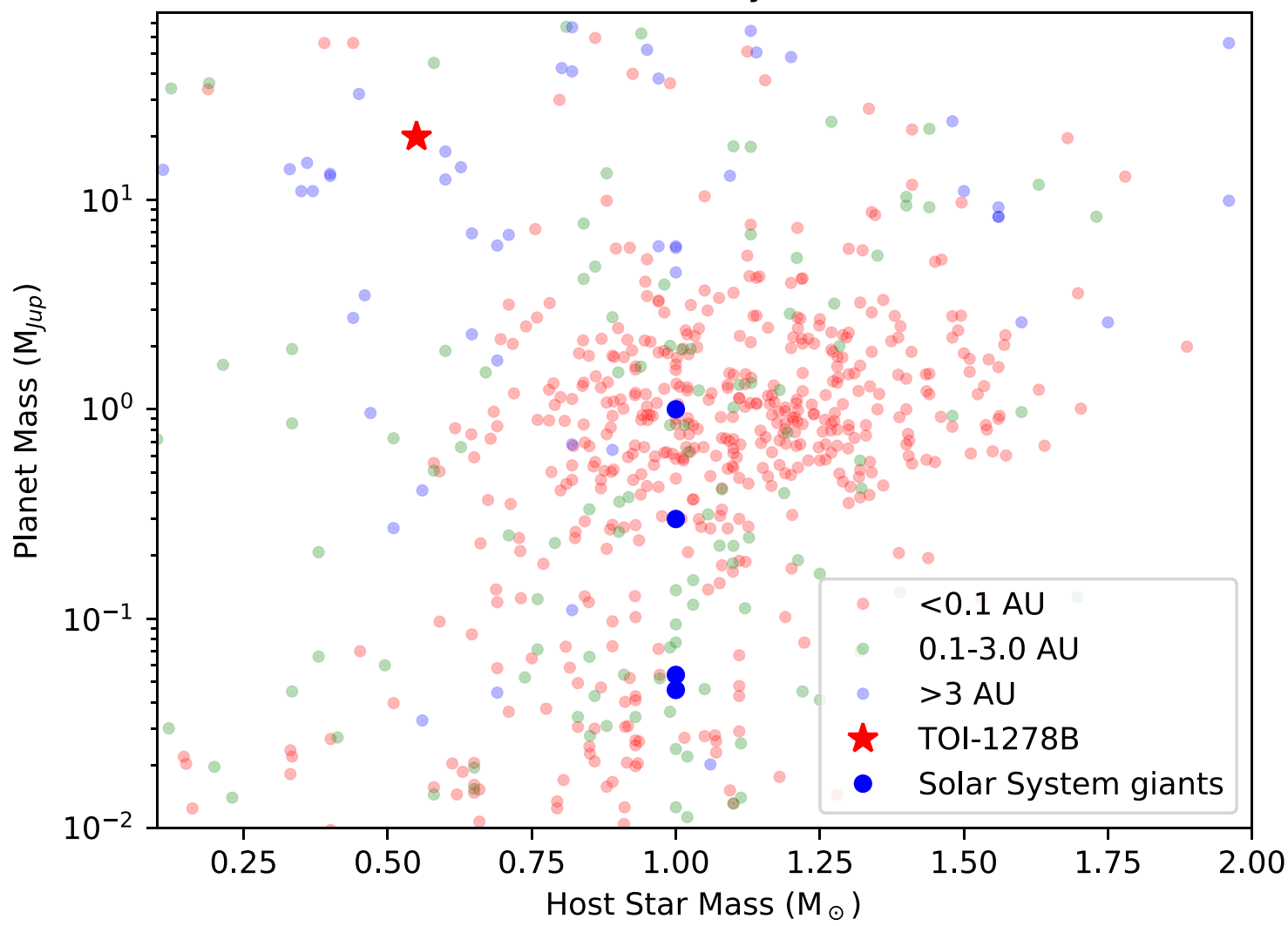

Figure 10. Substellar companion mass as a function of stellar host mass. Close-in companions (red dots) around $<0.5 M_{\odot}$ stars tend to be lighter than $0.1 M_{\text {Jup }}$. There are also a handful of massive ( $>40 M_{\text {Jup }}$ ) BDs in close-in orbits to M dwarfs. (Data from http://exoplanet.eu).

Table 4

Known 0.5-78 $M_{\text {Jup }}$ Companions to M Dwarfs with $P<100$ Days

\begin{tabular}{|c|c|c|c|c|c|}
\hline $\begin{array}{l}\text { Substellar } \\
\text { Companion }\end{array}$ & $\begin{array}{l}\text { Mass } \\
\left(M_{\text {Jup }}\right)\end{array}$ & SpT & $\begin{array}{r}\text { Period } \\
\text { (days) }\end{array}$ & References & Comment \\
\hline TOI-1899b & $0.66 \pm 0.07$ & M0 & 29.02 & Cañas et al. (2020) & Lowest-mass star known to host a transiting warm Jupiter \\
\hline NGTS-1b & $0.81 \pm 0.07$ & $\mathrm{M} 0.5 \pm 0.5$ & 2.65 & Bayliss et al. (2018) & Grazing transit, kinematically old host. \\
\hline GJ 876c & $0.760 \pm 0.002$ & M4 & 30.13 & $\begin{array}{l}\text { Marcy et al. (2001); } \\
\text { Trifonov et al. (2018) }\end{array}$ & $\begin{array}{l}\text { Non-transiting object, absolute mass estimated from dynamics } \\
\text { interaction by Correia et al. (2010) }\end{array}$ \\
\hline GJ 876b & $2.394 \pm 0.003$ & M4 & 61.08 & $\begin{array}{l}\text { Delfosse et al. (1998); } \\
\text { Trifonov et al. (2018) }\end{array}$ & Non-transiting object \\
\hline TOI-519 b & $<14$ & $\mathrm{M} 3.5_{-0.5}^{+1.0}$ & 1.26 & Parviainen et al. (2021) & $\begin{array}{l}\text { Upper limit on mass from light-curve analysis. } 1.07 R_{\text {Jup }} \text { consistent } \\
\text { with an object as light as } 0.5 M_{\text {Jup. }}\end{array}$ \\
\hline TOI-1278 B & $18.4 \pm 0.5$ & M0 & 14.48 & This work & \\
\hline $\mathrm{HD} 41004 \mathrm{Bb}$ & $18.4 \pm 0.22$ & M2 & 1.32 & Santos et al. (2002) & $\begin{array}{l}\text { Orbiting the } \mathrm{M} \text { component of a } \mathrm{K}+\mathrm{M} \text { stellar binary. Only } M \sin i \text { is } \\
\text { known }\end{array}$ \\
\hline NLTT 41135B & $33.7 \pm 2.8$ & M5.1 \pm 0.5 & 2.89 & Irwin et al. (2010) & Transiting one of the components of a 2 "! 3 binary. \\
\hline AD 3116B & $54.2 \pm 4.3$ & M3.9 & 1.98 & Gillen et al. (2017) & High-probability Praesepe member. \\
\hline RIK 72 B & $59.2 \pm 6.8$ & M2.5 & $\simeq 97.8$ & David et al. (2019) & Member of Upper Scorpius (5-7 Myr); highly inflated. \\
\hline TOI-263b & $61.6 \pm 4.0$ & M3.5 & 0.56 & Palle et al. (2021) & Orbit synchronized with the rotation of the star \\
\hline LHS6343C & $62.1 \pm 1.2$ & $\mathrm{M}^{\mathrm{b}}$ & 12.71 & $\begin{array}{l}\text { Johnson et al. (2011); } \\
\text { Montet et al. (2015) }\end{array}$ & Transiting LHS $6343 \mathrm{~A}$, itself member of a 0 "' 55 binary. \\
\hline LP 261-75B & $67.6 \pm 2.1$ & M4.5 & 1.88 & Irwin et al. (2018) & $10 \%$ deep transit at low impact parameter. \\
\hline NGTS-7Ab & $75_{-13.7}^{+3.0 \mathrm{a}}$ & M3 & 0.68 & Jackman et al. (2019) & $\begin{array}{l}\text { Young ( } 55 \mathrm{Myr} \text { ), active, tidally locked in a decaying orbit } \\
\text { (remaining lifetime of } 5-10 \mathrm{Myr} \text { ). Eclipsing one of the } \\
\text { components of a } 1 \text { "' } 13 \text { binary. }\end{array}$ \\
\hline
\end{tabular}

Notes.

${ }^{\mathrm{a}}$ The authors explore two scenarios regarding the distance of the NGTS-7 system, leading to significantly different companion masses. The GAIA EDR3 parallax $(6.33 \pm 0.07$ mas) for the host star is consistent with scenario $i$ and rules out the lighter mass of scenario $i i$.

${ }^{\mathrm{b}}$ Spectral subtype not specified, but $3431 \pm 21 \mathrm{~K}$ temperature (Montet et al. 2015) for host star corresponds to M2.5 \pm 0.5 (Boyajian et al. 2012). 

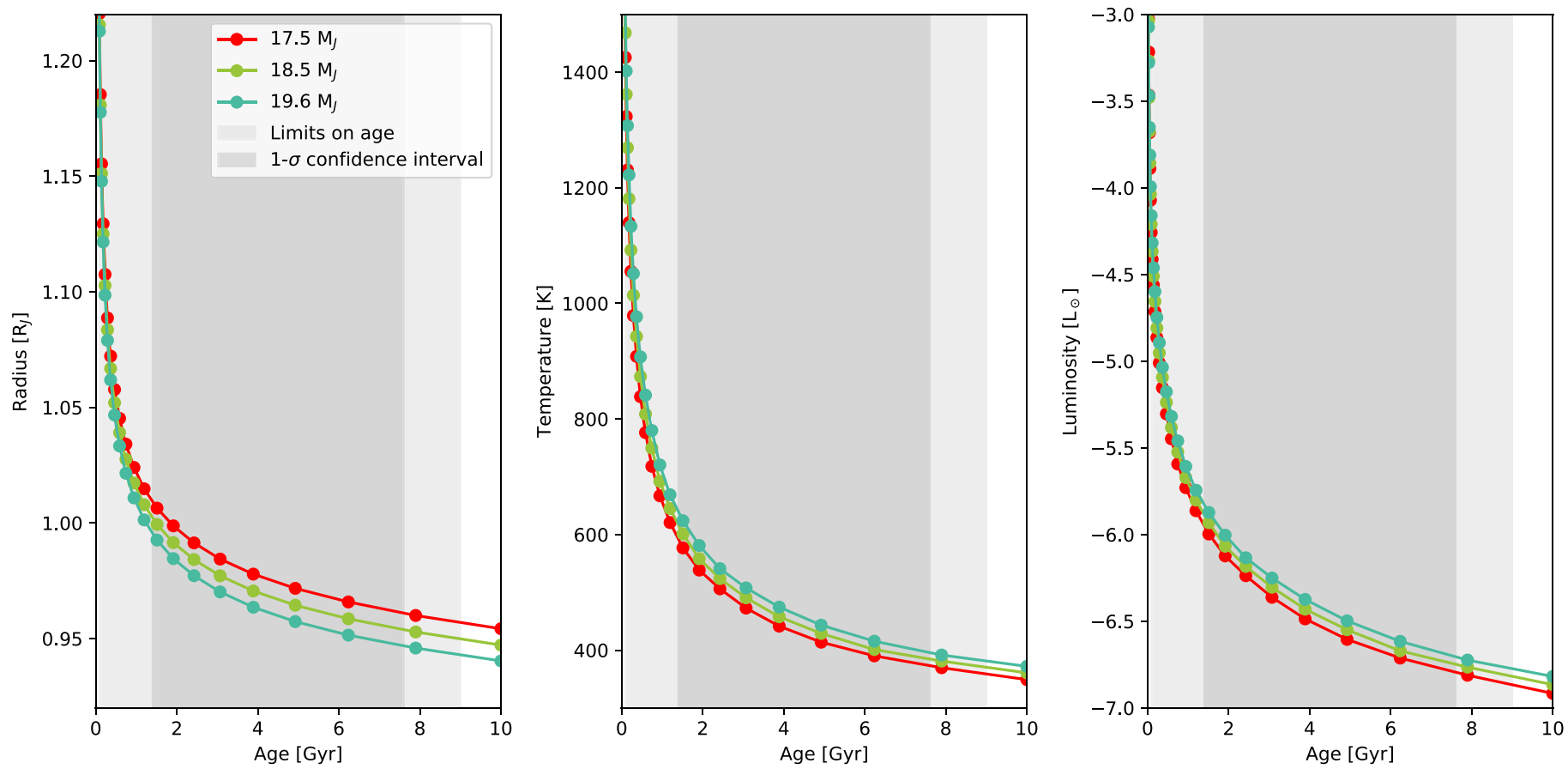

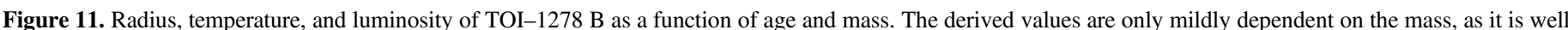

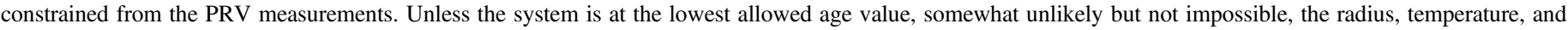
luminosity are constrained to within $\pm 2.5 \%, \pm 110 \mathrm{~K}$, and $\pm 0.4 \mathrm{dex}$, respectively.

Table 5

Model Properties of TOI-1278 B at Different Ages for a Mass of $18.5 M_{\mathrm{Jup}}$

\begin{tabular}{lccc}
\hline \hline & $0.1 \mathrm{Gyr}$ & $1.5 \mathrm{Gyr}$ & $7.9 \mathrm{Gyr}$ \\
\hline Temperature & $1362 \mathrm{~K}$ & $601 \mathrm{~K}$ & $381 \mathrm{~K}$ \\
Luminosity & $-4.37 \mathrm{~L} \odot$ & $-5.93 \mathrm{~L} \odot$ & $-6.76 \mathrm{~L} \odot$ \\
Gravity & 4.55 & 4.69 & 4.74 \\
Radius & $0.118 \mathrm{R}_{\odot}$ & $0.100 \mathrm{R}_{\odot}$ & $0.095 \mathrm{R}_{\odot}$ \\
$\Delta J_{M K O}$ & 6.878 & 10.992 & 15.285 \\
$\Delta H_{M K O}$ & 7.330 & 12.551 & 16.747 \\
$\Delta K_{M K O}$ & 7.640 & 13.113 & 18.720 \\
$\Delta W 1$ & 7.520 & 11.768 & 14.791 \\
$\Delta W 2$ & 6.028 & 8.275 & 9.953 \\
$\Delta W 3$ & 5.134 & 7.620 & 9.157 \\
\hline
\end{tabular}

Note. These values are interpolated from Phillips et al.'s (2020) BD models.

would be at the part-per-thousand level in the $K$ band and, most likely, significantly more challenging (See Table 5). Overall, the prospects for eclipse spectroscopy of TOI-1278 B are poor.

One can estimate the scale height of the atmosphere of TOI$1278 \mathrm{~B}$ and the corresponding transit-spectroscopy signal. As its atmosphere is most likely hydrogen-dominated, one can safely assume a mean-molecular weight of 2 AMU. Assuming a temperature of $380-1300 \mathrm{~K}$, which would correspond to the plausible age range, the scale height of the atmosphere would be $5-17 \mathrm{~km}$, making it an impractical target for transit spectroscopy.

The expected Rossiter-McLaughlin (RM) effect (Rossiter 1924; McLaughlin 1924) for this system can be estimated. Assuming a $v \sin i=1.1 \pm 0.9 \mathrm{~km} \mathrm{~s}^{-1}$ and a transit depth of $\sim 1 \%$, the maximum possible signal for the effect would be at the $7 \pm 6 \mathrm{~m} \mathrm{~s}^{-1}$ level. This is below the sensitivity of our SPIRou observations and would be challenging on such a faint target with current facilities. Considering that the transit is grazing, the signal is likely to be much smaller if the spin-orbit angle is close to $0^{\circ}$. A handful of hot Jupiters in polar orbits are known (e.g., Addison et al. 2013), but these orbit stars hotter than the Sun. Stars such as TOI-1278 have thicker convective layers that dampen orbital obliquity, suggesting that it is unlikely that this system displays a strong inclination and that its RM effect can be measured considering its transit configuration (Winn et al. 2010).

We note that TOI-1278 B is expected to be among the astrometric detections of substellar companions in Gaia. The semimajor axis of $0.1 \mathrm{au}$ and $75 \mathrm{pc}$ distance implies that the orbit subtends a 1.3 mas angle and that the astrometric orbital motion of the host star is $50 \mu$ as. At $G \sim 12.7$, the per-epoch astrometric accuracy is at the $34.2 \mu$ as level (Perryman et al. 2014). The presence of the companion most likely explains the astrometric_excess_noise_sig $=9.09$ value in the EDR3 catalog. This dimensionless parameter measures the significance of the excess in noise relative to the astrometric uncertainties when fitting a simple parallax models. For good fits of single stars, one would expect half of the targets to have a value of zero as the residuals to the fit will be (slightly) smaller than expected from uncertainties. The remaining targets will have values displaying a cumulative distribution corresponding to a Gaussian with a width of unity. Sources with a value above 2 are considered as likely having a significant noise excess in their parallax solutions. While Gaia does not have the proper time-sampling to probe short periods such as that of TOI-1278, the known orbital period from RV measurements could guide a determination of the actual astrometric orbit.

We dedicate this paper to the memory of France Allard, who until her untimely passing did so much to advance the modeling of $\mathrm{BD}$ atmospheres. 
Based on observations obtained at the Canada-FranceHawaii Telescope, which is operated from the summit of Maunakea by the National Research Council of Canada, the Institut National des Sciences de l'Univers of the Centre National de la Recherche Scientifique of France, and the University of Hawaii. The observations at the Canada-FranceHawaii Telescope were performed with care and respect from the summit of Maunakea, which is a significant cultural and historic site.

This work has been carried out within the framework of the National Centre of Competence in Research PlanetS supported by the Swiss National Science Foundation.

J.F.D. acknowledges funding from the European Research Council under the H2020 research \& innovation program (grant \#740651 NewWorlds).

This work was supported by FCT_-Fundação para a Ciência e a Tecnologia through national funds and by FEDER through COMPETE2020_Programa Operacional Competitividade e Internacionalização by these grants: UID/FIS/04434/2019; UIDB/04434/2020; UIDP/04434/2020; PTDC/FIS-AST/ 32113/2017 \& POCI-01-0145-FEDER-032113; PTDC/FISAST/28953/2017 \& POCI-01-0145-FEDER-028953.

J.H.C.M. is supported in the form of a work contract funded by Fundação para a Ciência e Tecnologia (FCT) with the reference DL 57/2016/CP1364/CT0007, and also supported from FCT through national funds and by FEDER-Fundo Europeu de Desenvolvimento Regional through COMPETE2020-Programa Operacional Competitividade e Internacionalização for these grants UIDB/04434/2020 \& UIDP/ 04434/2020, PTDC/FIS-AST/32113/2017 \& POCI-01-0145FEDER-032113, PTDC/FIS-AST/28953/2017 \& POCI-010145-FEDER-028953, PTDC/FIS-AST/29942/2017.

X.D., T.F., and I.B. received funding from the French Programme National de Physique Stellaire (PNPS) and the Programme National de Planétologie (PNP) of CNRS (INSU).

X.D., T.F., and I.B. acknowledge funding from the French National Research Agency (ANR) under contract No. ANR-18CE31-0019 (SPlaSH).

This work has made use of data from the European Space Agency (ESA) mission Gaia (https://www.cosmos.esa.int/ gaia), processed by the Gaia Data Processing and Analysis Consortium (DPAC, https://www.cosmos.esa.int/web/gaia/ dpac/consortium). Funding for the DPAC has been provided by national institutions, in particular the institutions participating in the Gaia Multilateral Agreement.

Funding for the TESS mission is provided by NASA's Science Mission directorate. We acknowledge the use of public TESS alert data from pipelines at the TESS Science Office and at the TESS Science Processing Operations Center. This research has made use of the Exoplanet Follow-up Observation Program website, which is operated by the California Institute of Technology, under contract with the National Aeronautics and Space Administration under the Exoplanet Exploration Program. This paper includes data collected by the TESS mission that are publicly available from the Mikulski Archive for Space Telescopes (MAST).

This research has made use of the SIMBAD database, operated at CDS, Strasbourg, France. This research has made use of NASA's Astrophysics Data System. This research made use of matplotlib, a Python library for publication quality graphics (Hunter 2007). This research made use of SciPy (SciPy 1.0 Contributors et al. 2020). This work made use of the IPython package (Perez \& Granger 2007). This research made use of Astropy, a community-developed core Python package for Astronomy (The Astropy Collaboration et al. 2013, 2018). This research made use of NumPy (Harris et al. 2020). This research made use of TOPCAT, an interactive graphical viewer and editor for tabular data (Taylor 2005). This research made use of Astroquery (Ginsburg et al. 2019). This research made use of ds 9, a tool for data visualization supported by the Chandra X-ray Science Center (CXC) and the High Energy Astrophysics Science Archive Center (HEASARC) with support from the JWST Mission office at the Space Telescope Science Institute for threedimensional visualization.

Facility: CFHT(SPIRou).

Software: astropy (Price-Whelan et al. 2018).

\section{Appendix \\ Table of SPIRou RV Measurements}

Table A1

SPIRou RV Measurements Binned per Epoch

\begin{tabular}{lcc}
\hline \hline $\begin{array}{l}\text { Time } \\
\text { BJD }\end{array}$ & $\begin{array}{c}\mathrm{RV} \\
{\left[\mathrm{m} \mathrm{s}^{-1}\right]}\end{array}$ & $\begin{array}{c}\sigma_{\mathrm{RV}} \\
{\left[\mathrm{m} \mathrm{s}^{-1}\right]}\end{array}$ \\
\hline 2459000.1000 & -28111.4 & 17.2 \\
2459003.0779 & -30822.7 & 28.5 \\
2459005.0466 & -31675.7 & 15.6 \\
2459006.0240 & -31491.6 & 14.9 \\
2459008.0282 & -29992.7 & 13.3 \\
2459008.9816 & -29051.9 & 18.3 \\
2459010.0464 & -28069.4 & 21.4 \\
2459011.1114 & -27299.0 & 21.5 \\
2459153.7576 & -29025.5 & 17.7 \\
2459154.7451 & -28075.8 & 15.9 \\
\hline
\end{tabular}




\section{ORCID iDs}

Étienne Artigau (ib https://orcid.org/0000-0003-3506-5667 Guillaume Hébrard (i) https://orcid.org/0000-0001-5450-7067 Neil J. Cook (iD https://orcid.org/0000-0003-4166-4121 Farbod Jahandar (iD https://orcid.org/0000-0003-0029-2835 Lison Malo (i) https://orcid.org/0000-0002-8786-8499 Jean-François Donati (iD https://orcid.org/0000-00015541-2887

Pía Cortés-Zuleta (iD https://orcid.org/0000-0002-6174-4666 Isabelle Boisse (i) https://orcid.org/0000-0001-8388-8399 Xavier Delfosse (10) https://orcid.org/0000-0001-5099-7978 Andres Carmona (iD https://orcid.org/0000-0003-2471-1299 Pascal Fouqué (iD https://orcid.org/0000-0002-1436-7351 Jason Rowe (1) https://orcid.org/0000-0002-5904-1865 Giuseppe Marino (10 https://orcid.org/0000-0001-8134-0389 Michael B. Lund (iD https://orcid.org/0000-0003-2527-1598 Luc Arnold (i) https://orcid.org/0000-0002-0111-1234 François Bouchy (Di) https://orcid.org/0000-0002-7613-393X Thierry Forveille (D) https://orcid.org/0000-0003-0536-4607 Nuno C. Santos (ib https://orcid.org/0000-0003-4422-2919 Xavier Bonfils (ib https://orcid.org/0000-0001-9003-8894 Pedro Figueira (1) https://orcid.org/0000-0001-8504-283X George Ricker (1) https://orcid.org/0000-0003-2058-6662 Sara Seager (ib https://orcid.org/0000-0002-6892-6948 Jon M. Jenkins (i) https://orcid.org/0000-0002-4715-9460 João Gomes da Silva (i) https://orcid.org/0000-00018056-9202

\section{References}

Addison, B. C., Tinney, C. G., Wright, D. J., et al. 2013, ApJL, 774, L9 Al-Rfou, R., Alain, G., Almahairi, A., et al. 2016, arXiv:1605.02688 Allard, F., Homeier, D., \& Freytag, B. 2010, arXiv:1011.5405 Allard, F., Homeier, D., \& Freytag, B. 2011, Proc. Int. Astronomical Union, 7,235

Anglada-Escudé, G., Amado, P. J., Barnes, J., et al. 2016, Natur, 536, 437 Ansdell, M., Williams, J. P., Marel, N. v. d., et al. 2016, ApJ, 828, 46 Artigau, É., Astudillo-Defru, N., Delfosse, X., et al. 2014, Proc. SPIE, 9149, 914905

Artigau, É., Malo, L., Doyon, R., et al. 2018b, AJ, 155, 198

Artigau, É., Saint-Antoine, J., Lévesque, P.-L., et al. 2018a, Proc. SPIE, 10709, 107091

Baraffe, I., Chabrier, G., Allard, F., \& Hauschildt, P. H. 1998, A\&A, 337, 403

Baroch, D., Morales, J. C., Ribas, I., et al. 2020, A\&A, 641, A69

Baron, F., Lafrenière, D., Artigau, E., et al. 2019, AJ, 158, 187

Bate, M. R. 2000, MNRAS, 314, 33

Bayliss, D., Gillen, E., Eigmüller, P., et al. 2018, MNRAS, 475, 4467

Bensby, T., Feltzing, S., \& Lundström, I. 2003, A\&A, 410, 527

Bergin, E. A., \& Williams, J. P. 2018, arXiv:1807.09631

Bertaux, J. L., Lallement, R., Ferron, S., Boonne, C., \& Bodichon, R. 2014, A\&A, 564, A46

Bonfils, X., Delfosse, X., Udry, S., et al. 2013, A\&A, 549, A109

Bouchy, F., Pepe, F., \& Queloz, D. 2001, A\&A, 374, 733

Boyajian, T. S., von Braun, K., van Belle, G., et al. 2012, ApJ, 757, 112

Cañas, C. I., Sferansson, G., Kanodia, S., et al. 2020, AJ, 160, 147

Carmichael, T. W., Quinn, S. N., Mustill, A. J., et al. 2020, AJ, 160, 53

Cersullo, F., Coffinet, A., Chazelas, B., Lovis, C., \& Pepe, F. 2019, A\&A, 624, A122

Claret, A. 2018, A\&A, 618, A20

Claret, A., \& Bloemen, S. 2011, A\&A, 529, A75

Collins, K. A., Kielkopf, J. F., Stassun, K. G., \& Hessman, F. V. 2017, AJ, 153,77

Correia, A. C. M., Couetdic, J., Laskar, J., et al. 2010, A\&A, 511, A21

Cushing, M. C., Rayner, J. T., \& Vacca, W. D. 2005, ApJ, 623, 1115

Cushing, M. C., Vacca, W. D., \& Rayner, J. T. 2004, PASP, 116, 362

Dai, D.-C., Li, Z., \& Stojkovic, D. 2018, arXiv:1812.04674

David, T. J., Hillenbrand, L. A., Gillen, E., et al. 2019, ApJ, 872, 161

Deacon, N. R., Schlieder, J. E., \& Murphy, S. J. 2016, MNRAS, 457, 3191 del Peloso, E. F., da Silva, L., Porto de Mello, G. F., \& Arany-Prado, L. I. 2005, A\&A, 440, 1153

Delfosse, X., Forveille, T., Mayor, M., et al. 1998, arXiv:astro-ph/9808026 Delorme, P., Gagné, J., Girard, J. H., et al. 2013, A\&A, 553, L5

Demory, B.-O., Ségransan, D., Forveille, T., et al. 2009, A\&A, 505, 205

Donati, J.-F., Kouach, D., Lacombe, M., et al. 2018, Spirou: A NIR Spectropolarimeter/High-Precision Velocimeter for the CFHT (Berlin: Springer), 107

Donati, J.-F., Kouach, D., Moutou, C., et al. 2020, MNRAS, 498, 5684

Donati, J.-F., Semel, M., Carter, B. D., Rees, D. E., \& Cameron, A. C. 1997, MNRAS, 291, 658

Eastman, J., Gaudi, B. S., \& Agol, E. 2013, PASP, 125, 83

Einstein, A. 1917, Sitzungsberichte der Königlich Preußischen Akademie der Wissenschaften (Berlin), 142

Faherty, J. K., Riedel, A. R., Cruz, K. L., et al. 2016, ApJS, 225, 10

Figueira, P., Adibekyan, V. Z., Oshagh, M., et al. 2016, A\&A, 586, A101

Filippazzo, J. C., Rice, E. L., Faherty, J., et al. 2015, ApJ, 810, 158

Flewelling, H. A., Magnier, E. A., Chambers, K. C., et al. 2020, ApJS, 251, 62

Foreman-Mackey, D., Luger, R., Czekala, I., et al. 2020, exoplanet-dev/ exoplanet: exoplanet v0.4.3, Zenodo, doi:10.5281/ZENODO.1998447

Frasca, A., Biazzo, K., Alcalá, J. M., et al. 2017, A\&A, 602, A33

Gagné, J., Mamajek, E. E., Malo, L., et al. 2018, ApJ, 856, 23

Gaia Collaboration, Smart, R. L., Sarro, L. M., et al. 2021, A\&A, 649, A6

Gillen, E., Hillenbrand, L. A., David, T. J., et al. 2017, ApJ, 849, 11

Gillon, M., Triaud, A. H. M. J., Demory, B.-O., et al. 2017, Natur, 542, 456

Ginsburg, A., Sipőcz, B. M., Brasseur, C. E., et al. 2019, AJ, 157, 98

Goldreich, P., \& Soter, S. 1966, Icar, 5, 375

Harris, C. R., Millman, K. J., van der Walt, S. J., et al. 2020, Natur, 585, 357

Heller, R., Jackson, B., Barnes, R., Greenberg, R., \& Homeier, D. 2010, A\&A, 514, A22

Hobson, M. J., Bouchy, F., Cook, N. J., et al. 2021, A\&A, 648, A48

Horne, K. 1986, PASP, 98, 609

Hunter, J. D. 2007, CSE, 9, 90

Husser, T.-O., Wende-von Berg, S., Dreizler, S., et al. 2013, A\&A, 553, A6

Irwin, J., Buchhave, L., Berta, Z. K., et al. 2010, ApJ, 718, 1353

Irwin, J., Charbonneau, D., Nutzman, P., et al. 2009, IAU Symp. 253 , Transiting Planets, ed. F. Pont, D. Sasselov, \& M. J. Holman, (Cambridge: Cambridge Univ. Press), 37

Irwin, J. M., Charbonneau, D., Esquerdo, G. A., et al. 2018, AJ, 156, 140

Itoh, Y., Hayashi, M., Tamura, M., et al. 2005, ApJ, 620, 984

Jackman, J. A. G., Wheatley, P. J., Bayliss, D., et al. 2019, MNRAS, 489, 5146

Jenkins, J. M., Twicken, J. D., McCauliff, S., et al. 2016, Proc. SPIE, 9913, 99133E

Jensen, E. 2013, Tapir: A web interface for transit/eclipse observability, Astrophysics Source Code Library, record ascl:1306.007

Johnson, J. A., Apps, K., Gazak, J. Z., et al. 2011, ApJ, 730, 79

Kanodia, S., \& Wright, J. 2018, RNAAS, 2, 4

Kirkpatrick, J. D., Martin, E. C., Smart, R. L., et al. 2019, ApJS, 240, 19

Kirkpatrick, J. D., Schneider, A., Fajardo-Acosta, S., et al. 2014, AJ, 783, 122

Kupka, F. G., Ryabchikova, T. A., Piskunov, N. E., Stempels, H. C., \& Weiss, W. W. 2000, OAst, 9, 590

Löhner-Böttcher, J., Schmidt, W., Schlichenmaier, R., Steinmetz, T., \& Holzwarth, R. 2019, A\&A, 624, A57

Li, J., Tenenbaum, P., Twicken, J. D., et al. 2019, PASP, 131, 024506

Luger, R., Agol, E., Foreman-Mackey, D., et al. 2019, AJ, 157, 64

Luhman, K. L., Wilson, J. C., Brandner, W., et al. 2006, ApJ, 649, 894

Malo, L., Doyon, R., Feiden, G. A., et al. 2014, ApJ, 792, 37

Marcy, G. W., Butler, R. P., Fischer, D., et al. 2001, ApJ, 556, 296

Martioli, E., Hébrard, G., Moutou, C., et al. 2020, A\&A, 641, L1

McLaughlin, D. B. 1924, ApJ, 60, 22

Meunier, N., Mignon, L., \& Lagrange, A.-M. 2017, A\&A, 607, A124

Meyer, M. R., Amara, A., Reggiani, M., \& Quanz, S. P. 2018, A\&A, 612, L3

Micheau, Y., Challita, Z., Gallou, G., et al. 2018, Proc. SPIE, 10702, 210

Mireles, I., Shporer, A., Grieves, N., et al. 2020, AJ, 160, 133

Montet, B. T., Johnson, J. A., Muirhead, P. S., et al. 2015, ApJ, 800, 134

Muirhead, P. S., Dressing, C. D., Mann, A. W., et al. 2018, AJ, 155, 180

Naud, M.-E., Artigau, E., Malo, L., et al. 2014, ApJ, 787, 5

Palle, E., Luque, R., Zapatero Osorio, M. R., et al. 2021, AJ, 650, 55

Parviainen, H., Palle, E., Zapatero-Osorio, M. R., et al. 2021, A\&A, 646, A16 Patra, K. C., Winn, J. N., Holman, M. J., et al. 2017, AJ, 154, 4

Pecaut, M. J., \& Mamajek, E. E. 2013, ApJS, 208, 9

Perez, F., \& Granger, B. E. 2007, CSE, 9, 21

Perryman, M., Hartman, J., Bakos, G. A., \& Lindegren, L. 2014, ApJ, 797, 14 Phillips, M. W., Tremblin, P., Baraffe, I., et al. 2020, A\&A, 637, A38

Piskunov, N. E., Kupka, F., Ryabchikova, T. A., Weiss, W. W., \& Jeffery, C. S. 1995, A\&AS, 112, 525 
Price-Whelan, A. M., Sipőcz, B. M., Günther, H. M., et al. 2018, AJ, 156, 123

Rayner, J. T., Cushing, M. C., \& Vacca, W. D. 2009, ApJS, 185, 289

Rayner, J. T., Toomey, D. W., Onaka, P. M., et al. 2003, PASP, 115, 362

Reiners, A., Zechmeister, M., Caballero, J. A., et al. 2018, A\&A, 612, A49

Ricker, G. R., Winn, J. N., Vanderspek, R., et al. 2014, JATIS, 1, 014003

Rossiter, R. A. 1924, ApJ, 60, 15

Ryabchikova, T., Piskunov, N., Kurucz, R. L., et al. 2015, PhyS, 90, 054005

Salvatier, J., Wiecki, T., \& Fonnesbeck, C. 2016, Astrophysics Source Code Library, record ascl:1610.016

Santos, N. C., Mayor, M., Naef, D., et al. 2002, A\&A, 392, 215

SciPy 1.0 Contributors, Virtanen, P., Gommers, R., et al. 2020, Nature Methods, 17, 261

Skrutskie, M. F., Cutri, R. M., Stiening, R., et al. 2006, AJ, 131, 1163

Smith, J. C., Stumpe, M. C., Van Cleve, J. E., et al. 2012, PASP, 124, 1000

Stassun, K. G., Oelkers, R. J., Paegert, M., et al. 2019, AJ, 158, 138

Stassun, K. G., Oelkers, R. J., Pepper, J., et al. 2018, AJ, 156, 102

Stetson, P. 2000, PASP, 112, 925
Stumpe, M. C., Smith, J. C., Catanzarite, J. H., et al. 2014, PASP, 126, 100 Stumpe, M. C., Smith, J. C., Van Cleve, J. E., et al. 2012, PASP, 124, 985

Taylor, M. B. 2005, in Proc. Conf. held 24-27 October, 2004 in Pasadena, 347, ed. P. Shopbell, M. Britton, \& R. Ebert (San Francisco, CA: SP), 29

The Astropy Collaboration, Price-Whelan, A. M., Sipőcz, B. M., et al. 2018, AJ, 156,123

The Astropy Collaboration, Robitaille, T. P., Tollerud, E. J., et al. 2013, A\&A, 558, A33

Trifonov, T., Kürster, M., Zechmeister, M., et al. 2018, A\&A, 609, A117

Twicken, J. D., Catanzarite, J. H., Clarke, B. D., et al. 2018, PASP, 130, 064502

Vacca, W., Cushing, M., \& Rayner, J. 2003, PASP, 115, 389

Veeder, G. J. 1974, AJ, 79, 1056

Wang, J., Fischer, D. A., Horch, E. P., \& Huang, X. 2015, ApJ, 799, 229

Winn, J. N., Fabrycky, D., Albrecht, S., \& Johnson, J. A. 2010, ApJL, 718, L145 